\title{
Convex Relaxation of Optimal Power Flow Part I: Formulations and Equivalence
}

\author{
Steven H. Low \\ Electrical Engineering, Computing+Mathematical Sciences \\ Engineering and Applied Science, Caltech \\ slow@caltech.edu
}

April 15, 2014

\begin{abstract}
This tutorial summarizes recent advances in the convex relaxation of the optimal power flow (OPF) problem, focusing on structural properties rather than algorithms. Part I presents two power flow models, formulates OPF and their relaxations in each model, and proves equivalence relations among them. Part II presents sufficient conditions under which the convex relaxations are exact.
\end{abstract}

Citation: IEEE Transactions on Control of Network Systems, 15(1): 15-27, March 2014. This is an extended version with Appendices VIII and IX that provide some mathematical preliminaries and proofs of the main results. All proofs can be found in their original papers. We provide proofs here because (i) it is convenient to have all proofs in one place and in a uniform notation, and (ii) some of the formulations and presentations here are slightly different from those in the original papers.

A preliminary and abridged version has appeared in Proceedings of the IREP Symposium - Bulk Power System Dynamics and Control - IX, Rethymnon, Greece, August 25-30, 2013. 


\section{CONTENTS}

I Introduction $\quad 4$

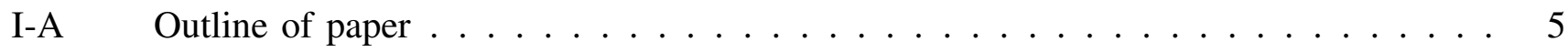

I-B Notations ........................ 5

II Power flow models $\quad 6$

II-A Bus injection model ...................... 6

II-B Branch flow model . . . . . . . . . . . . . . . . . . 7

II-C Equivalence ........................ 7

$\begin{array}{ll}\text { III Optimal power flow } & 8\end{array}$

III-A Bus injection model ..................... 8

III-B Branch flow model . . . . . . . . . . . . . . . . . . 9

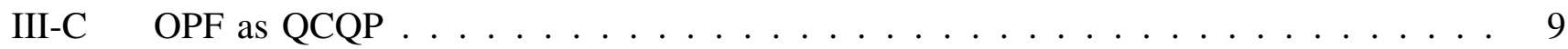

IV Feasible sets and relaxations: BIM 11

IV-A Preliminaries . ........................ 11

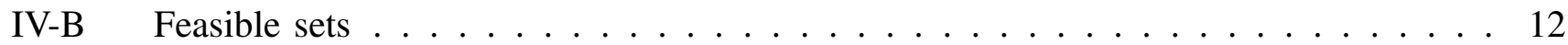

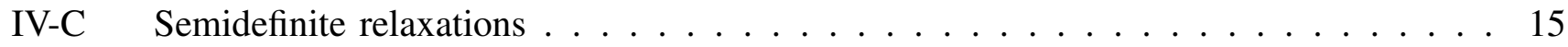

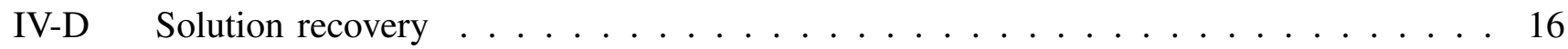

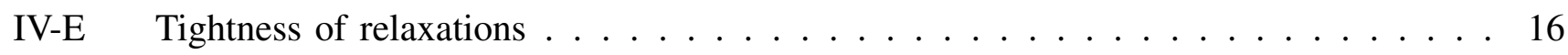

IV-F Chordal relaxation . . . . . . . . . . . . . . . . . . . . 17

$\begin{array}{llr}\text { V Feasible sets and relaxations: BFM } & 17\end{array}$

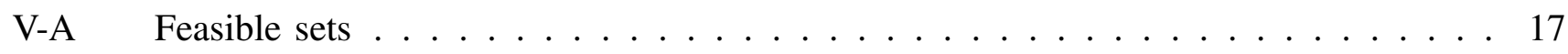

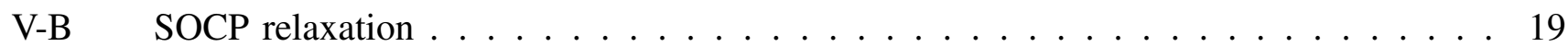

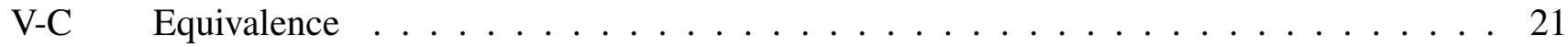

$\begin{array}{llr}\text { VI BFM for radial networks } & 21\end{array}$

VI-A Recursive equations and graph orientation ................ 21

VI-B Linear approximation and bounds ................... 23

$\begin{array}{lr}\text { VII Conclusion } & 25\end{array}$

Appendix: VIII: Mathematical preliminaries 26

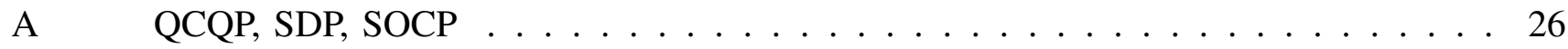

B Graph, partial matrix and completion .................. 28

C Chordal relaxation . . . . . . . . . . . . . . . . . . 29 
Appendix: IX: Proofs of main results $\quad 32$

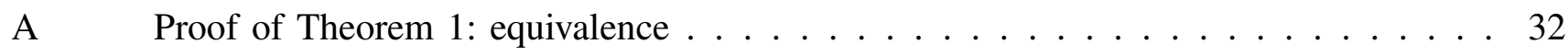

B Proof of Theorem 2: rank-1 characterization . . . . . . . . . . . . . 33

C Proof of Corollary 3: uniqueness of completion . . . . . . . . . . . . . 34

D Proof of Theorem 5: BIM feasible sets . . . . . . . . . . . . . . 34

E Proof of Theorem 7: BFM feasible sets . . . . . . . . . . . . 34

F Proof of Theorem 8: BFM cycle condition . . . . . . . . . . . 36

G Proof of Theorem 9: radial networks . . . . . . . . . . . . . . . 37

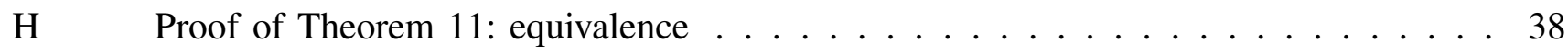

I Proof of Lemma 12: voltage bound . . . . . . . . . . . . . . . 40

$\begin{array}{ll}\text { References } & 42\end{array}$

Acknowledgment. We thank the support of NSF through NetSE CNS 0911041, ARPA-E through GENI DE-AR0000226, Southern California Edison, the National Science Council of Taiwan through NSC 1033113-P-008-001, the Los Alamos National Lab (DoE), and Caltech's Resnick Institute. 


\section{INTRODUCTION}

For our purposes an optimal power flow (OPF) problem is a mathematical program that seeks to minimize a certain function, such as total power loss, generation cost or user disutility, subject to the Kirchhoff's laws as well as capacity, stability and security constraints. OPF is fundamental in power system operations as it underlies many applications such as economic dispatch, unit commitment, state estimation, stability and reliability assessment, volt/var control, demand response, etc. There has been a great deal of research on OPF since Carpentier's first formulation in 1962 [1]. An early solution appears in [2] and extensive surveys can be found in e.g. [3], [4], [5], [6], [7], [8], [9], [10], [11], [12], [13], [14].

Power flow equations are quadratic and hence OPF can be formulated as a quadratically constrained quadratic program (QCQP). It is generally nonconvex and hence NP-hard. A large number of optimization algorithms and relaxations have been proposed. A popular approximation is a linear program, called DC OPF, obtained through the linearization of the power flow equations e.g. [15], [16], [17], [18], [19]. See also [20] for a more accurate linear approximation. To the best of our knowledge solving OPF through semidefinite relaxation is first proposed in [21] as a second-order cone program (SOCP) for radial (tree) networks and in [22] as a semidefinite program (SDP) for general networks in a bus injection model. It is first proposed in [23], [24] as an SOCP for radial networks in the branch flow model of [25], [26]. See Remark 6 below for more details. While these convex relaxations have been illustrated numerically in [21] and [22], whether or when they will turn out to be exact is first studied in [27]. Exploiting graph sparsity to simplify the SDP relaxation of OPF is first proposed in [28], [29] and analyzed in [30], [31].

Convex relaxation of quadratic programs has been applied to many engineering problems; see e.g. [32]. There is a rich theory and extensive empirical experiences. Compared with other approaches, solving OPF through convex relaxation offers several advantages. First, while DC OPF is useful in a wide variety of applications, it is not applicable in other applications; see Remark 10. Second a solution of DC OPF may not be feasible (may not satisfy the nonlinear power flow equations). In this case an operator may tighten some constraints in DC OPF and solve again. This may not only reduce efficiency but also relies on heuristics that are hard to scale to larger systems or faster control in the future. Third, when they converge, most nonlinear algorithms compute a local optimal usually without assurance on the quality of the solution. In contrast a convex relaxation provides for the first time the ability to check if a solution is globally optimal. If it is not, the solution provides a lower bound on the minimum cost and hence a bound on how far any feasible solution is from optimality. Unlike approximations, if a relaxed problem is infeasible, it is a certificate that the original OPF is infeasible.

This two-part tutorial explains the main theoretical results on semidefinite relaxations of OPF developed in the last few years. Part I presents two power flow models that are useful in different situations, formulates OPF and its convex relaxations in each model, and clarifies their relationship. Part II [33] presents sufficient conditions that guarantee the relaxations are exact, i.e. when one can recover a globally optimal solution of OPF from an optimal solution of its relaxations. We focus on basic results using the simplest OPF formulation and does not cover many relevant works in the literature, such as stochastic OPF e.g. [34], [35], [36], distributed OPF e.g. [37], [38], [39], [40], [41], [42], new applications e.g. [43], [44], or what to do when relaxation fails e.g. [45], [46], [47], to name just a few. 


\section{A. Outline of paper}

Many mathematical models have been used to model power networks. In Part I of this two-part paper we present two such models, we call the bus injection model (BIM) and the branch flow model (BFM). Each model consists of a set of power flow equations. Each models a power network in that the solutions of each set of equations, called the power flow solutions, describe the steady state of the network. We prove that these two models are equivalent in the sense that there is a bijection between their solution sets (Section II). We formulate OPF within each model where the power flow solutions define the feasible set of OPF (Section III). Even though BIM and BFM are equivalent some results are much easier to formulate or prove in one model than the other; see Remark 2 in Section II.

The complexity of OPF formulated here lies in the nonconvexity of power flow equations that gives rise to a nonconvex feasible set of OPF. We develop various characterizations of the feasible set and design convex supersets based on these characterizations. Different designs lead to different convex relaxations and we prove their relationship (Sections IV and V). When a relaxation is exact an optimal solution of the original nonconvex OPF can be recovered from any optimal solution of the relaxation. In Part II [33] we present sufficient conditions that guarantee the exactness of convex relaxations.

Branch flow models are originally proposed for networks with a tree topology, called radial networks, e.g. [25], [26], [48], [49], [50], [51], [23], [52], [53]. They take a recursive structure that simplifies the computation of power flow solutions, e.g. [54], [55], [48]. The model of [25], [26] also has a linearization that offers several advantages over DC OPF in BIM; see Remark 10. The linear approximation provides simple bounds on the branch powers and voltage magnitudes in the nonlinear BFM (Section VI). These bounds are used in [56] to prove a sufficient condition for exact relaxation.

We make algorithmic recommendations in Section VII based on the results presented here.

This extended version differs from the journal version only in the addition of two Appendices. Appendix VIII provides some mathematical preliminaries and Appendix IX proofs of all main results. Even though all proofs can be found in their original papers, we provide proofs here because (i) it is convenient to have all proofs in one place and in a uniform notation, and (ii) some of the formulations and presentations here are slightly different from those in the original papers.

\section{B. Notations}

Let $\mathbb{C}$ denote the set of complex numbers, $\mathbb{R}$ the set of real numbers, and $\mathbb{N}$ the set of integers. For $a \in \mathbb{C}, \operatorname{Re} a$ and $\operatorname{Im} a$ denote the real and imaginary parts of $a$ respectively. For any set $A \subseteq \mathbb{C}^{n}, \operatorname{conv} A$ denotes the convex hull of $A$. For $a \in \mathbb{R},[a]^{+}:=\max \{a, 0\}$. For $a, b \in \mathbb{C}, a \leq b$ means $\operatorname{Re} a \leq \operatorname{Re} b$ and $\operatorname{Im} a \leq \operatorname{Im} b$. We abuse notation to use the same symbol $a$ to denote either a complex number $\operatorname{Re} a+\mathbf{i} \operatorname{Im} a$ or a 2 -dimensional real vector $a=(\operatorname{Re} a, \operatorname{Im} a)$ depending on the context.

In general scalar or vector variables are in small letters, e.g. $u, w, x, y, z$. Most power system quantities however are in capital letters, e.g. $S_{j k}, P_{j k}, Q_{j k}, I_{j}, V_{j}$. A variable without a subscript denotes a vector with appropriate components, e.g. $s:=\left(s_{j}, j=0, \ldots, n\right), S:=\left(S_{j k},(j, k) \in E\right)$. For vectors $x, y, x \leq y$ denotes componentwise inequality.

Matrices are usually in capital letters. The transpose of a matrix $A$ is denoted by $A^{T}$ and its Hermitian (complex conjugate) transpose by $A^{H}$. A matrix $A$ is Hermitian if $A=A^{H}$. $A$ is positive semidefinite (or 
psd), denoted by $A \succeq 0$, if $A$ is Hermitian and $x^{H} A x \geq 0$ for all $x \in \mathbb{C}^{n}$; in particular if $A \succeq 0$ then by definition $A=A^{H}$. For matrices $A, B, A \succeq B$ means $A-B$ is psd. Let $\mathbb{S}^{n}$ be the set of all $n \times n$ Hermitian matrices and $\mathbb{S}_{+}^{n}$ the set of $n \times n$ psd matrices.

A graph $G=(N, E)$ consists of a set $N$ of nodes and a set $E \subseteq N \times N$ of edges. If $G$ is undirected then $(j, k) \in E$ if and only if $(k, j) \in E$. If $G$ is directed then $(j, k) \in E$ only if $(k, j) \notin E$; in this case we will use $(j, k)$ and $j \rightarrow k$ interchangeably to denote an edge pointing from $j$ to $k$. We sometimes use $\tilde{G}=(N, \tilde{E})$ to denote a directed graph. By " $j \sim k$ " we mean an edge $(j, k)$ if $G$ is undirected and either $j \rightarrow k$ or $k \rightarrow j$ if $G$ is directed. Sometimes we write $j \in G$ or $(j, k) \in G$ to mean $j \in N$ or $(j, k) \in E$ respectively. A cycle $c:=\left(j_{1}, \ldots, j_{K}\right)$ is an ordered set of nodes $j_{k} \in N$ so that $\left(j_{k}, j_{k+1}\right) \in E$ for $k=1, \ldots, K$ with the understanding that $j_{K+1}:=j_{1}$. In that case we refer to a link or a node in the cycle by $\left(j_{k}, j_{k+1}\right) \in c$ or $j_{k} \in c$ respectively.

\section{POWER FlOW MODELS}

In this section we describe two mathematical models of power networks and prove their equivalence. By a "mathematical model" we mean a set of variables and a set of equations relating these variables. These equations are motivated by the physical system, but mathematically, they are the starting point from which all claims are derived.

\section{A. Bus injection model}

Consider a power network modeled by a connected undirected graph $G\left(N^{+}, E\right)$ where $N^{+}:=\{0\} \cup N$, $N:=\{1,2, \ldots, n\}$, and $E \subseteq N^{+} \times N^{+}$. Each node in $N^{+}$represents a bus and each edge in $E$ represents a transmission or distribution line. We use "bus" and "node" interchangeably and "line" and "edge" interchangeably. For each edge $(i, j) \in E$ let $y_{i j} \in \mathbb{C}$ be its admittance. A bus $j \in N^{+}$can have a generator, a load, both or neither. Let $V_{j}$ be the complex voltage at bus $j \in N^{+}$and $\left|V_{j}\right|$ denote its magnitude. Bus 0 is the slack bus. Its voltage is fixed and we assume without loss of generality that $V_{0}=1 \angle 0^{\circ}$ per unit (pu). Let $s_{j}$ be the net complex power injection (generation minus load) at bus $j \in N^{+}$.

The bus injection model (BIM) is defined by the following power flow equations that describe the Kirchhoff's laws:

$$
s_{j}=\sum_{k: j \sim k} y_{j k}^{H} V_{j}\left(V_{j}^{H}-V_{k}^{H}\right), \quad j \in N^{+}
$$

Let the set of power flow solutions $V$ for each $s$ be:

$$
\mathbb{V}(s):=\left\{V \in \mathbb{C}^{n+1} \mid V \text { satisfies }(1)\right\}
$$

For convenience we include $V_{0}$ in the vector variable $V:=\left(V_{j}, j \in N^{+}\right)$with the understanding that $V_{0}:=$ $1 \angle 0^{\circ}$ is fixed.

Remark 1: Bus types. Each bus $j$ is characterized by two complex variables $V_{j}$ and $s_{j}$, or equivalently, four real variables. The buses are usually classified into three types, depending on which two of the four real variables are specified. For the slack bus $0, V_{0}$ is given and $s_{0}$ is variable. For a generator bus (also called $P V$-bus), $\operatorname{Re}\left(s_{j}\right)=p_{j}$ and $\left|V_{j}\right|$ are specified and $\operatorname{Im}\left(s_{j}\right)=q_{j}$ and $\angle V_{j}$ are variable. For a load bus 
(also called $P Q$-bus), $s_{j}$ is specified and $V_{j}$ is variable. The power flow or load flow problem is: given two of the four real variables specified for each bus, solve the $n+1$ complex equations in (1) for the remaining $2(n+1)$ real variables. For instance when all $n$ buses $j \neq 0$ are all load buses, the power flow problem solves (1) for the $n$ complex voltages $V_{j}, j \neq 0$, and the power injection $s_{0}$ at the slack bus 0 . This can model a distribution system with a substation at bus 0 and $n$ constant-power loads at the other buses. For optimal power flow problems $p_{j}$ and $\left|V_{j}\right|$ on generator buses or $s_{j}$ on load buses can be variables as well. For instance economic dispatch optimizes real power generations $p_{j}$ at generator buses; demand response optimizes demands $s_{j}$ at load buses; and volt/var control optimizes reactive powers $q_{j}$ at capacitor banks, tap changers, or inverters. These remarks also apply to the branch flow model presented next.

\section{B. Branch flow model}

In the branch flow model we adopt a connected directed graph $\tilde{G}=\left(N^{+}, \tilde{E}\right)$ where each node in $N^{+}:=$ $\{0,1, \ldots, n\}$ represents a bus and each edge in $\tilde{E} \subseteq N^{+} \times N^{+}$represents a transmission or distribution line. Fix an arbitrary orientation for $\tilde{G}$ and let $m:=|\tilde{E}|$ be the number of directed edges in $\tilde{G}$. Denote an edge by $(j, k)$ or $j \rightarrow k$ if it points from node $j$ to node $k$. For each edge $(j, k) \in \tilde{E}$ let $z_{j k}:=1 / y_{j k}$ be the complex impedance on the line; let $I_{j k}$ be the complex current and $S_{j k}=P_{j k}+\mathbf{i} Q_{j k}$ be the sending-end complex power from buses $j$ to $k$. For each bus $j \in N^{+}$let $V_{j}$ be the complex voltage at bus $j$. Assume without loss of generality that $V_{0}=1 \angle 0^{\circ} \mathrm{pu}$. Let $s_{j}$ be the net complex power injection at bus $j$.

The branch flow model (BFM) in [24] is defined by the following set of power flow equations:

$$
\begin{aligned}
\sum_{k: j \rightarrow k} S_{j k} & =\sum_{i: i \rightarrow j}\left(S_{i j}-z_{i j}\left|I_{i j}\right|^{2}\right)+s_{j}, j \in N^{+} \\
I_{j k} & =y_{j k}\left(V_{j}-V_{k}\right), \quad j \rightarrow k \in \tilde{E} \\
S_{j k} & =V_{j} I_{j k}^{H}, \quad j \rightarrow k \in \tilde{E}
\end{aligned}
$$

where (2b) is the Ohm's law, (2c) defines branch power, and (2a) imposes power balance at each bus. The quantity $z_{i j}\left|I_{i j}\right|^{2}$ represents line loss so that $S_{i j}-z_{i j}\left|I_{i j}\right|^{2}$ is the receiving-end complex power at bus $j$ from bus $i$.

Let the set of solutions $\tilde{x}:=(S, I, V)$ of BFM for each $s$ be:

$$
\tilde{\mathbb{X}}(s):=\left\{\tilde{x} \in \mathbb{C}^{2 m+n+1} \mid \tilde{x} \text { satisfies }(2)\right\}
$$

For convenience we include $V_{0}$ in the vector variable $V:=\left(V_{j}, j \in N^{+}\right)$with the understanding that $V_{0}:=$ $1 \angle 0^{\circ}$ is fixed.

\section{Equivalence}

Even though the bus injection model (1) and the branch flow model (2) are defined by different sets of equations in terms of their own variables, both are models of the Kirchhoff's laws and therefore must be related. We now clarify the precise sense in which these two mathematical models are equivalent. We say two sets $A$ and $B$ are equivalent, denoted by $A \equiv B$, if there is a bijection between them [57].

Theorem 1: $\mathbb{V}(s) \equiv \tilde{\mathbb{X}}(s)$ for any power injections $s$. 
Remark 2: Two models. Given the bijection between the solution sets $\mathbb{V}(s)$ and $\tilde{\mathbb{X}}(s)$ any result in one model is in principle derivable in the other. Some results however are much easier to state or derive in one model than the other. For instance BIM, which is widely used in transmission network problems, allows a much cleaner formulation of the semidefinite program (SDP) relaxation. BFM for radial networks has a convenient recursive structure that allows a more efficient computation of power flows and leads to a useful linear approximation of BFM; see Section VI. The sufficient condition for exact relaxation in [56] provides intricate insights on power flows that are hard to formulate or prove in BIM. Finally, since BFM directly models branch flows $S_{j k}$ and currents $I_{j k}$, it is easier to use for some applications. We will therefore freely use either model depending on which is more convenient for the problem at hand.

\section{OPTIMAL POWER FLOW}

\section{A. Bus injection model}

As mentioned in Remark 1 an optimal power flow problem optimizes both variables $V$ and $s$ over the solution set of the BIM (1). In addition all voltage magnitudes must satisfy:

$$
\underline{v}_{j} \leq\left|V_{j}\right|^{2} \leq \bar{v}_{j}, \quad j \in N^{+}
$$

where $\underline{v}_{j}$ and $\bar{v}_{j}$ are given lower and upper bounds on voltage magnitudes. Throughout this paper we assume $\underline{v}_{j}>0$ to avoid triviality. The power injections are also constrained:

$$
\underline{s}_{j} \leq s_{j} \leq \bar{s}_{j}, \quad j \in N^{+}
$$

where $\underline{s}_{j}$ and $\bar{s}_{j}$ are given bounds on the injections at buses $j$.

Remark 3: OPF constraints. If there is no bound on the load or on the generation at bus $j$ then $\underline{s}_{j}=$ $-\infty-\mathbf{i} \infty$ or $\bar{s}_{j}=\infty+\mathbf{i} \infty$ respectively. On the other hand (4) also allows the case where $s_{j}$ is fixed (e.g. a constant-power load), by setting $\underline{s}_{j}=\bar{s}_{j}$ to the specified value. For the slack bus 0 , unless otherwise specified, we always assume $\underline{v}_{0}=\bar{v}_{0}=1$ and $\underline{s}_{0}=-\infty-\mathbf{i} \infty, \bar{s}_{0}=\infty+\mathbf{i} \infty$. Therefore we sometimes replace $j \in N^{+}$in (3) and (4) by $j \in N$.

We can eliminate the variables $s_{j}$ from the OPF formulation by combining (1) and (4) into

$$
\underline{s}_{j} \leq \sum_{k:(j, k) \in E} y_{j k}^{H} V_{j}\left(V_{j}^{H}-V_{k}^{H}\right) \leq \bar{s}_{j}, j \in N^{+}
$$

Then OPF in the bus injection model can be defined just in terms of the complex voltage vector $V$. Define

$$
\mathbb{V}:=\left\{V \in \mathbb{C}^{n+1} \mid V \text { satisfies (3),(5) }\right\}
$$

$\mathbb{V}$ is the feasible set of optimal power flow problems in BIM.

Let the cost function be $C(V)$. Typical costs include the cost of generating real power at each generator bus or line loss over the network. All these costs can be expressed as functions of $V$. Then the problem of interest is:

\section{OPF:}

$$
\min _{V} C(V) \quad \text { subject to } \quad V \in \mathbb{V}
$$


Since (5) is quadratic, $\mathbb{V}$ is generally a nonconvex set. OPF is thus a nonconvex problem and NP-hard to solve in general.

\section{B. Branch flow model}

Denote the variables in the branch flow model (2) by $\tilde{x}:=(S, I, V, s) \in \mathbb{C}^{2(m+n+1)}$. We can also eliminate the variables $s_{j}$ as for the bus injection model by combining (2a) and (4) but it will prove convenient to retain $s:=\left(s_{j}, j \in N^{+}\right)$as part of the variables. Define the feasible set in the branch flow model:

$$
\tilde{\mathbb{X}}:=\left\{\tilde{x} \in \mathbb{C}^{2(m+n+1)} \mid \tilde{x} \text { satisfies }(2),(3),(4)\right\}
$$

Let the cost function in the branch flow model be $C(\tilde{x})$. Then the optimal power flow problem in the branch flow model is:

\section{OPF:}

$$
\min _{\tilde{x}} C(\tilde{x}) \quad \text { subject to } \quad \tilde{x} \in \tilde{\mathbb{X}}
$$

Since (2) is quadratic, $\mathbb{X}$ is generally a nonconvex set. OPF is thus a nonconvex problem and NP-hard to solve in general.

Remark 4: OPF equivalence. By Theorem 1 there is a bijection between $\mathbb{V}$ and $\tilde{\mathbb{X}}$. Throughout this paper we assume that the cost functions in BIM and BFM are equivalent under this bijection and we abuse notation to denote them by the same symbol $C(\cdot)$. Then OPF (7) in BIM and (9) in BFM are equivalent.

Remark 5: OPF variants. OPF as defined in (7) and (9) is a simplified version that ignores other important constraints such as line limits, security constraints, stability constraints, and chance constraints; see extensive surveys in [3], [4], [5], [6], [7], [8], [9], [10], [11], [12], [13], [14], [58], [59] and a recent discussion in [60] on real-life OPF problems. Some of these can be incorporated without any change to the results in this paper (e.g. see [24], [61] for models that include shunt elements and line limits). Indeed a shunt element $y_{j}$ at bus $j$ can be easily included in BIM by modifying (1) into:

$$
s_{j}=\sum_{k: j \sim k} y_{j k}^{H} V_{j}\left(V_{j}^{H}-V_{k}^{H}\right)+y_{j}^{H}\left|V_{j}\right|^{2}
$$

or included in BFM by modifying (2a) into:

$$
\sum_{k: j \rightarrow k} S_{j k}+y_{j}^{H}\left|V_{j}\right|^{2}=\sum_{i: i \rightarrow j}\left(S_{i j}-z_{i j}\left|I_{i j}\right|^{2}\right)+s_{j}
$$

\section{C. $O P F$ as $Q C Q P$}

Before we describe convex relaxations of OPF we first show that, when $C(V):=V^{H} C V$ is quadratic in $V$ for some Hermitian matrix $C, \mathrm{OPF}$ is indeed a quadratically constrained quadratic program (QCQP) by converting it into the standard form. We will use the derivation in [61] for OPF (7) in BIM. OPF (9) in BFM can similarly be converted into a standard form QCQP. 
Define the $(n+1) \times(n+1)$ admittance matrix $Y$ by

$$
Y_{i j}= \begin{cases}\sum_{k: k \sim i} y_{i k}, & \text { if } i=j \\ -y_{i j}, & \text { if } i \neq j \text { and } i \sim j \\ 0 & \text { otherwise }\end{cases}
$$

$Y$ is symmetric but not necessarily Hermitian. Let $I_{j}$ be the net injection current from bus $j$ to the rest of the network. Then the current vector $I$ and the voltage vector $V$ are related by the Ohm's law $I=Y V$. BIM (1) is equivalent to:

$$
s_{j}=V_{j} I_{j}^{H}=\left(e_{j}^{H} V\right)\left(I^{H} e_{j}\right)
$$

where $e_{j}$ is the $(n+1)$-dimensional vector with 1 in the $j$ th entry and 0 elsewhere. Hence, since $I=Y V$, we have

$$
s_{j}=\operatorname{tr}\left(e_{j}^{H} V V^{H} Y^{H} e_{j}\right)=\operatorname{tr}\left(Y^{H} e_{j} e_{j}^{H}\right) V V^{H}=V^{H} Y_{j}^{H} V
$$

where $Y_{j}:=e_{j} e_{j}^{H} Y$ is an $(n+1) \times(n+1)$ matrix with its $j$ th row equal to the $j$ th row of the admittance matrix $Y$ and all other rows equal to the zero vector. $Y_{j}$ is in general not Hermitian so that $V^{H} Y_{j}^{H} V$ is in general a complex number. Its real and imaginary parts can be expressed in terms of the Hermitian and skew Hermitian components of $Y_{j}^{H}$ defined as:

$$
\Phi_{j}:=\frac{1}{2}\left(Y_{j}^{H}+Y_{j}\right) \text { and } \Psi_{j}:=\frac{1}{2 \mathbf{i}}\left(Y_{j}^{H}-Y_{j}\right)
$$

Then

$$
\operatorname{Re} s_{j}=V^{H} \Phi_{j} V \text { and } \operatorname{Im} s_{j}=V^{H} \Psi_{j} V
$$

Let their upper and lower bounds be denoted by

$$
\begin{aligned}
& \underline{p}_{j}:=\operatorname{Re} \underline{s}_{j} \quad \text { and } \quad \bar{p}_{j}:=\operatorname{Re} \bar{s}_{j} \\
& \underline{q}_{j}:=\operatorname{Re} \underline{s}_{j} \quad \text { and } \quad \bar{q}_{j}:=\operatorname{Re} \bar{s}_{j}
\end{aligned}
$$

Let $J_{j}:=e_{j} e_{j}^{H}$ denote the Hermitian matrix with a single 1 in the $(j, j)$ th entry and 0 everywhere else. Then OPF (7) can be written as a standard form QCQP:

$$
\begin{aligned}
\min _{V \in \mathbb{C}^{n+1}} & V^{H} C V \\
\text { subject to } & V^{H} \Phi_{j} V \leq \bar{p}_{j}, V^{H}\left(-\Phi_{j}\right) V \leq-\underline{p}_{j} \\
& V^{H} \Psi_{j} V \leq \bar{q}_{j}, V^{H}\left(-\Psi_{j}\right) V \leq-\underline{q}_{j} \\
& V^{H} J_{j} V \leq \bar{v}_{j}, V^{H}\left(-J_{j}\right) V \leq-\underline{v}_{j}
\end{aligned}
$$

where $j \in N^{+}$in (10). 


\section{FEASIBLE SETS AND RELAXATIONS: BIM}

In this and the next section we derive semidefinite relaxations of OPF and clarify their relations. The cost function $C$ of OPF is usually assumed to be convex in its variables. The difficulty of OPF formulated here thus arises from the nonconvex feasible sets $\mathbb{V}$ for BIM and $\tilde{\mathbb{X}}$ for BFM. The basic approach to deriving convex relaxations of OPF is to design convex supersets of (equivalent sets of) $\mathbb{V}$ or $\tilde{\mathbb{X}}$ and minimize the same cost function over these supersets. Different choices of convex supersets lead to different relaxations, but they all provide a lower bound to OPF. If every optimal solution of a convex relaxation happens to lie in $\mathbb{V}$ or $\tilde{\mathbb{X}}$ then it is also feasible and hence optimal for the original OPF. In this case we say the realxation is exact.

In this section we present three characterizations of the feasible set $\mathbb{V}$ in BIM. These characterizations naturally suggest convex supersets and semidefinite relaxations of OPF, and we prove equivalence relations among them. In the next section we treat BFM. In Part II of the paper we discuss sufficient conditions that guaranteed exact relaxations.

\section{A. Preliminaries}

Since OPF is a nonconvex QCQP there is a standard semidefinite relaxation through the equivalence relation: for any Hermitian matrix $M, V^{H} M V=\operatorname{tr} M V V^{H}=\operatorname{tr} M W$ for a psd rank-1 matrix $W$. Applying this transformation to the QCQP formulation (10) leads to an equivalent problem of the form:

$$
\begin{array}{cl}
\min _{W \in \mathbb{S}^{n+1}} & \operatorname{tr} C W \\
\text { subject to } & \operatorname{tr} C_{l} W \leq b_{l}, W \succeq 0, \text { rank } W=1
\end{array}
$$

for appropriate Hermitian matrices $C_{l}$ and real numbers $b_{l}$. This problem is equivalent to (10) because given a psd rank-1 solution $W$, a unique solution $V$ of (10) can be recovered through rank-1 factorization $W=V V^{H}$. Unlike (10) which is quadratic in $V$ this problem is convex in $W$ except the nonconvex rank-1 constraint. Removing the rank-1 constraint yields the standard SDP relaxation.

We now generalize this intuition to characterize the feasible set $\mathbb{V}$ in (6) in terms of partial matrices. These characterizations lead naturally to SDP, chordal, and second-order cone program (SOCP) relaxations of OPF in BIM, as shown in [57], [31].

We start with some basic definitions on partial matrices and their completions; see e.g. [62], [63], [64] for more details. Fix any connected undirected graph $F$ with $n$ vertices and $m$ edges connecting distinct vertices. ${ }^{1}$ A partial matrix $W_{F}$ is a set of $2 m+n$ complex numbers defined on $F$ :

$$
W_{F}:=\left\{\left[W_{F}\right]_{j j},\left[W_{F}\right]_{j k},\left[W_{F}\right]_{k j} \mid \text { nodes } j \text { and edges }(j, k) \text { of } F\right\}
$$

$W_{F}$ can be interpreted as a matrix with entries partially specified by these complex numbers. If $F$ is a complete graph (in which there is an edge between every pair of vertices) then $W_{F}$ is a fully specified $n \times n$ matrix. A completion $W$ of $W_{F}$ is any fully specified $n \times n$ matrix that agrees with $W_{F}$ on graph $F$, i.e.,

$$
[W]_{j j}=\left[W_{F}\right]_{j j},[W]_{j k}=\left[W_{F}\right]_{j k} \text { for } j,(j, k) \in F
$$

\footnotetext{
${ }^{1}$ In this subsection we abuse notation and use $n, m$ to denote general integers unrelated to the number of buses or lines in a power network.
} 
Given an $n \times n$ matrix $W$ we use $W_{F}$ to denote the submatrix of $W$ on $F$, i.e., the partial matrix consisting of the entries of $W$ defined on graph $F$. If $q$ is a clique (a fully connected subgraph) of $F$ then let $W_{F}(q)$ denote the fully-specified principal submatrix of $W_{F}$ defined on $q$. We extend the definitions of Hermitian, psd, and rank-1 for matrices to partial matrices, as follows. A partial matrix $W_{F}$ is Hermitian, denoted by $W_{F}=W_{F}^{H}$, if $\left[W_{F}\right]_{j k}=\left[W_{F}\right]_{k j}^{H}$ for all $(j, k) \in F$; it is $p s d$, denoted by $W_{F} \succeq 0$, if $W_{F}$ is Hermitian and the principal submatrices $W_{F}(q)$ are psd for all cliques $q$ of $F$; it is rank-1, denoted by rank $W_{F}=1$, if the principal submatrices $W_{F}(q)$ are rank-1 for all cliques $q$ of $F$. We say $W_{F}$ is $2 \times 2$ psd (rank-1) if, for all edges $(j, k) \in F$, the $2 \times 2$ principal submatrices

$$
W_{F}(j, k):=\left[\begin{array}{ll}
{\left[W_{F}\right]_{j j}} & {\left[W_{F}\right]_{j k}} \\
{\left[W_{F}\right]_{k j}} & {\left[W_{F}\right]_{k k}}
\end{array}\right]
$$

are psd (rank-1), denoted by $W_{F}(j, k) \succeq 0$ (rank $\left.W_{F}(j, k)=1\right) . F$ is a chordal graph if either $F$ has no cycle or all its minimal cycles (ones without chords) are of length three. A chordal extension $c(F)$ of $F$ is a chordal graph that contains $F$, i.e., $c(F)$ has the same vertex set as $F$ but an edge set that is a superset of $F$ 's edge set. In that case we call the partial matrix $W_{c(F)}$ a chordal extension of the partial matrix $W_{F}$. Every graph $F$ has a chordal extension, generally nonunique. In particular a complete supergraph of $F$ is a trivial chordal extension of $F$.

For our purposes chordal graphs are important because of the result [62, Theorem 7] that every psd partial matrix has a psd completion if and only if the underlying graph is chordal. When a positive definite completion exists, there is a unique positive definite completion, in the class of all positive definite completions, whose determinant is maximal. Theorem 2 below extends this to rank-1 partial matrices.

\section{B. Feasible sets}

We can now characterize the feasible set $\mathbb{V}$ of OPF defined in (6). Recall the undirected connected graph $G=\left(N^{+}, E\right)$ that models a power network. Given a voltage vector $V \in \mathbb{V}$ define a partial matrix $W_{G}:=W_{G}(V)$ : for $j \in N^{+}$and $(j, k) \in E$,

$$
\begin{aligned}
{\left[W_{G}\right]_{j j}:=\left|V_{j}\right|^{2} } \\
{\left[W_{G}\right]_{j k}:=V_{j} V_{k}^{H}=:\left[W_{G}\right]_{k j}^{H} }
\end{aligned}
$$

Then the constraints (5) and (3) imply that the partial matrix $W_{G}$ satisfies ${ }^{2}$

$$
\begin{gathered}
\underline{s}_{j} \leq \sum_{k:(j, k) \in E} y_{j k}^{H}\left(\left[W_{G}\right]_{j j}-\left[W_{G}\right]_{j k}\right) \leq \bar{s}_{j}, \quad j \in N^{+} \\
\underline{v}_{j} \leq\left[W_{G}\right]_{j j} \leq \bar{v}_{j},
\end{gathered}
$$

\footnotetext{
${ }^{2}$ The constraint (12a) can also be written compactly in terms of the admittance matrix $Y$ as in [65]:
}

$$
\underline{s} \leq \operatorname{diag}\left(W Y^{H}\right) \leq \bar{s}
$$


Following Section III-C these constraints can also be written in a (partial) matrix form as:

$$
\begin{aligned}
& \underline{p}_{j} \leq \operatorname{tr} \Phi_{j} W_{G} \leq \bar{p}_{j} \\
& \underline{q}_{j} \leq \operatorname{tr} \Psi_{j} W_{G} \leq \bar{q}_{j} \\
& \underline{v}_{j} \leq \operatorname{tr} J_{j} W_{G} \leq \bar{v}_{j}
\end{aligned}
$$

The converse is not always true: given a partial matrix $W_{G}$ that satisfies (12) it is not always possible to recover a voltage vector $V$ in $\mathbb{V}$. Indeed this is possible if and only if $W_{G}$ has a completion $W$ that is psd rank-1, because in that case $W$ satisfies (12) since $y_{j k}=0$ if $(j, k) \notin E$ and it can be uniquely factored as $W=V V^{H}$ with $V \in \mathbb{V}$. We hence seek conditions additional to (12) on the partial matrix $W_{G}$ that guarantee that it has a psd rank-1 completion $W$ from which $V \in \mathbb{V}$ can be recovered. Our first key result provides such a characterization.

We say that a partial matrix $W_{G}$ satisfies the cycle condition if for every cycle $c$ in $G$

$$
\sum_{(j, k) \in c} \angle\left[W_{G}\right]_{j k}=0 \quad \bmod 2 \pi
$$

When $\angle\left[W_{G}\right]_{j k}$ represent voltage phase differences across each line then the cycle condition imposes that they sum to zero $(\bmod 2 \pi)$ around any cycle. The next theorem, proved in [57, Theorem 3] and [31], implies that $W_{G}$ has a psd rank-1 completion $W$ if and only if $W_{G}$ is $2 \times 2$ psd rank-1 on $G$ and satisfies the cycle condition (13), if and only if it has a chordal extension $W_{c(G)}$ that is psd rank-1. ${ }^{3}$

Consider the following conditions on $(n+1) \times(n+1)$ matrices $W$ and partial matrices $W_{c(G)}$ and $W_{G}$ :

$$
\begin{aligned}
W & \succeq 0, \quad \operatorname{rank} W=1 \\
W_{c(G)} & \succeq 0, \quad \operatorname{rank} W_{c(G)}=1 \\
W_{G}(j, k) & \succeq 0, \quad \operatorname{rank} W_{G}(j, k)=1, \quad(j, k) \in E,
\end{aligned}
$$

Theorem 2: Fix a graph $G$ on $n+1$ nodes and any chordal extension $c(G)$ of $G$. Assuming $W_{j j}>0$, $\left[W_{c(G)}\right]_{j j}>0$ and $\left[W_{G}\right]_{j j}>0, j \in N^{+}$, we have:

(1) Given an $(n+1) \times(n+1)$ matrix $W$ that satisfies (14), its submatrix $W_{c(G)}$ satisfies (15).

(2) Given a partial matrix $W_{c(G)}$ that satisfies (15), its submatrix $W_{G}$ satisfies (16) and the cycle condition (13).

(3) Given a partial matrix $W_{G}$ that satisfies (16) and the cycle condition (13), there is a completion $W$ of $W_{G}$ that satisfies (14).

Informally Theorem 2 says that (14) is equivalent to (15) is equivalent to (16)+(13). It characterizes a property of the full matrix $W$ (rank $W=1$ ) in terms of its submatrices $W_{c(G)}$ and $W_{G}$. This is important because the submatrices are typically much smaller than $W$ for large sparse networks and much easier to compute. The theorem thus allows us to solve simpler problems in terms of partial matrices as we now explain.

\footnotetext{
${ }^{3}$ The theorem also holds with psd replaced by negative semidefinite.
} 
Define the set of Hermitian matrices:

$$
\mathbb{W}:=\left\{W \in \mathbb{S}^{n+1} \mid W \text { satisfies }(12),(14)\right\}
$$

Fix any chordal extension $c(G)$ of $G$ and define the set of Hermitian partial matrices $W_{c(G)}$ :

$$
\mathbb{W}_{c(G)}:=\left\{W_{c(G)} \mid W_{c(G)} \text { satisfies }(12),(15)\right\}
$$

Finally define the set of Hermitian partial matrices $W_{G}$ :

$$
\mathbb{W}_{G}:=\left\{W_{G} \mid W_{G} \text { satisfies (12),(13),(16) }\right\}
$$

Note that the definition of psd for partial matrices implies that $W_{c(G)}$ and $W_{G}$ are Hermitian. The assumption $\underline{v}_{j}>0, j \in N^{+}$implies that all matrices or partial matrices have strictly positive diagonal entries.

Theorem 2 implies that given a partial matrix $W_{c(G)} \in \mathbb{W}_{c(G)}$ or a partial matrix $W_{G} \in \mathbb{W}_{G}$ there is a psd rank-1 completion $W \in \mathbb{W}$ from which a solution $V \in \mathbb{V}$ of OPF can be recovered. In fact we know more: given any Hermitian partial matrix $W_{G}$ (not necessarily in $\mathbb{W}_{G}$ ), the set of all completions of $W_{G}$ that satisfies the condition in Theorem 2(3) consists of a single psd rank-1 matrix and infinitely many indefinite non-rank-1 matrices; see [57, Theorems 5 and 8] and discussions therein. Hence the psd rank-1 completion $W$ of a $W_{G} \in \mathbb{W}_{G}$ is unique.

Corollary 3: Given a partial matrix $W_{c(G)} \in \mathbb{W}_{c(G)}$ or $W_{G} \in \mathbb{W}_{G}$ there is a unique psd rank-1 completion $W \in \mathbb{W}$.

Recall that two sets $A$ and $B$ are equivalent $(A \equiv B)$ if there is a bijection between them. Even though $\mathbb{W}, \mathbb{W}_{c(G)}, \mathbb{W}_{G}$ are different kinds of spaces Theorem 2 and Corollary 3 imply that they are all equivalent to the feasible set of OPF.

Theorem 4: $\mathbb{V} \equiv \mathbb{W} \equiv \mathbb{W}_{c(G)} \equiv \mathbb{W}_{G}$.

Theorem 4 suggests three equivalent problems to OPF. We assume the cost function $C(V)$ in OPF depends on $V$ only through the partial matrix $W_{G}$ defined in (11). For example if the cost is total real line loss in the network then $C(V)=\sum_{j} \operatorname{Re} s_{j}=\sum_{j} \sum_{k:(j, k) \in E} \operatorname{Re}\left(\left[W_{G}\right]_{j j}-\left[W_{G}\right]_{j k}\right) y_{j k}^{H}$. If the cost is a weighted sum of real generation power then $C(V)=\sum_{j}\left(c_{j} \operatorname{Re} s_{j}+p_{j}^{d}\right)$ where $p_{j}^{d}$ are the given real power demands at buses $j$; again $C(V)$ is a function of the partial matrix $W_{G}$. Then Theorem 4 implies that OPF (7) is equivalent to

$$
\min _{W} C\left(W_{G}\right) \quad \text { subject to } W \in \hat{\mathbb{W}}
$$

where $\mathbb{W}$ is any one of the sets $\mathbb{W}, \mathbb{W}_{c(G)}, \mathbb{W}_{G}$. Specifically, given an optimal solution $W^{\text {opt }}$ in $\mathbb{W}$, it can be uniquely decomposed into $W^{\mathrm{opt}}=V^{\mathrm{opt}}\left(V^{\mathrm{opt}}\right)^{H}$. Then $V^{\mathrm{opt}}$ is in $\mathbb{V}$ and an optimal solution of OPF (7). Alternatively given an optimal solution $W_{F}^{\text {opt }}$ in $\mathbb{W}_{c(G)}$ or $\mathbb{W}_{G}$, Corollary 3 guarantees that $W_{F}^{\text {opt }}$ has a unique psd rank-1 completion $W^{\text {opt }}$ in $\mathbb{W}$ from which an optimal $V^{\text {opt }} \in \mathbb{V}$ can be recovered. In fact given a partial matrix $W_{G} \in \mathbb{W}_{G}\left(\right.$ or $W_{c(G)} \in \mathbb{W}_{c(G)}$ ) there is a more direct construction of a feasible solution $V \in \mathbb{V}$ of OPF than through its completion; see Section IV-D. 


\section{Semidefinite relaxations}

Hence solving OPF (7) is equivalent to solving (20) over any of $\mathbb{W}, \mathbb{W}_{c(G)}, \mathbb{W}_{G}$ for an appropriate matrix variable. The difficulty with solving (20) is that the feasible sets $\mathbb{W}, \mathbb{W}_{c(G)}$, and $\mathbb{W}_{G}$ are still nonconvex due to the rank-1 constraints and the cycle condition (13). Their removal leads to SDP, chordal, and SOCP relaxations of OPF respectively.

Relax $\mathbb{W}, \mathbb{W}_{c(G)}$ and $\mathbb{W}_{G}$ to the following convex supersets:

$$
\begin{aligned}
\mathbb{W}^{+} & :=\left\{W \in \mathbb{S}^{n+1} \mid W_{G} \text { satisfies }(12), W \succeq 0\right\} \\
\mathbb{W}_{c(G)}^{+} & :=\left\{W_{c(G)} \mid W_{G} \text { satisfies }(12), W_{c(G)} \succeq 0\right\} \\
\mathbb{W}_{G}^{+} & :=\left\{W_{G} \mid W_{G} \text { satisfies }(12), W_{G}(j, k) \succeq 0,(j, k) \in E\right\}
\end{aligned}
$$

Define the problems:

\section{OPF-sdp:}

$$
\min _{W} C\left(W_{G}\right) \quad \text { subject to } \quad W \in \mathbb{W}^{+}
$$

OPF-ch:

$$
\min _{W_{c(G)}} C\left(W_{G}\right) \quad \text { subject to } \quad W_{c(G)} \in \mathbb{W}_{c(G)}^{+}
$$

\section{OPF-socp:}

$$
\min _{W_{G}} C\left(W_{G}\right) \quad \text { subject to } \quad W_{G} \in \mathbb{W}_{G}^{+}
$$

The condition $W_{G}(j, k) \succeq 0$ in the definition of $\mathbb{W}_{G}^{+}$is equivalent to $\left[W_{G}\right]_{j k}=\left[W_{G}\right]_{k j}^{H}$ and (recall the assumption $\underline{v}_{j}>0, j \in N^{+}$)

$$
\left[W_{G}\right] j j>0,\left[W_{G}\right]_{k k}>0,\left[W_{G}\right]_{j j}\left[W_{G}\right]_{k k} \geq\left|\left[W_{G}\right]_{j k}\right|^{2}
$$

This is a second-order cone and hence OPF-socp is indeed an SOCP in the rotated form.

Remark 6: Literature. SOCP relaxation for OPF seems to be first proposed in [21] for the bus injection model (1), and in [23], [24] for the branch flow model (2) as explained in the next section. By defining a new set of variables $v_{j}:=\left|V_{j}\right|^{2}, R_{j k}:=\left|V_{j}\right|\left|V_{k}\right| \cos \left(\theta_{j}-\theta_{k}\right)$, and $I_{j k}:=\left|V_{j}\right|\left|V_{k}\right| \sin \left(\theta_{j}-\theta_{k}\right)$ where $\theta_{j}:=\angle V_{j}$, [21] rewrites the bus injection model (1) in the complex domain as a set of linear equations in these new variables in the real domain and the following quadratic equations:

$$
v_{j} v_{k}=R_{j k}^{2}+I_{j k}^{2}
$$

Relaxing these equalities to $v_{j} v_{k} \geq R_{j k}^{2}+I_{j k}^{2}$ enlarges the solution set to a second-order cone that is equivalent to $\mathbb{W}_{G}^{+}$in this paper. SDP relaxation is first proposed in [22] for the bus injection model and analyzed in [27]. Chordal relaxation for OPF is first proposed in [28], [29] and analyzed in [30], [31]. 


\section{Solution recovery}

When the convex relaxations OPF-sdp, OPF-ch, OPF-socp are exact, i.e., if their optimal solutions $W^{\text {sdp }}$, $W_{c h}^{\text {ch }}, W_{G}^{\text {socp }}$ happen to lie in $\mathbb{W}, \mathbb{W}_{c(G)}, \mathbb{W}_{G}$ respectively, then an optimal solution $V^{\text {opt }}$ of the original OPF can be recovered from these solutions. Indeed the recovery method works not just for an optimal solution, but any feasible solution that lies in $\mathbb{W}, \mathbb{W}_{c(G)}$ or $\mathbb{W}_{G}$. Moreover, given a $W \in \mathbb{W}$ or a $W_{c(G)} \in \mathbb{W}_{c(G)}$, the construction of $V$ depends on $W$ or $W_{c(G)}$ only through their submatrix $W_{G}$. We hence describe the method for recovering the unique $V$ from a $W_{G}$, which may be a partial matrix in $\mathbb{W}_{G}$ or the submatrix of a (partial) matrix in $\mathbb{W}$ or $\mathbb{W}_{c(G)}$.

Let $T$ be an arbitrary spanning tree of $G$ rooted at bus 0 . Let $\mathbb{P}_{j}$ denote the unique path from node 0 to node $j$ in $T$. Recall that $V_{0}=1 \angle 0^{\circ}$ without loss of generality. For $j=1, \ldots, n$, let

$$
\begin{aligned}
\left|V_{j}\right| & :=\sqrt{\left[W_{G}\right]_{j j}} \\
\angle V_{j} & :=-\sum_{(i, k) \in \mathbb{P}_{j}} \angle\left[W_{G}\right]_{i k}
\end{aligned}
$$

Then it can be checked that $V$ is in (6) and feasible for OPF.

\section{E. Tightness of relaxations}

Since $\mathbb{W} \subseteq \mathbb{W}^{+}, \mathbb{W}_{c(G)} \subseteq \mathbb{W}_{c(G)}^{+}, \mathbb{W}_{G} \subseteq \mathbb{W}_{G}^{+}$, the relaxations OPF-sdp, OPF-ch, OPF-socp all provide lower bounds on OPF (7) in light of Theorem 4. OPF-socp is the simplest computationally. OPF-ch usually requires more computation than OPF-socp but much less than OPF-sdp for large sparse networks (even though OPF-ch can be as complex as OPF-sdp in the worse case [63], [64]). The relative tightness of the relaxations depends on the network topology. For a general mesh network OPF-sdp is as tight a relaxation as OPF-ch and they are strictly tighter than OPF-socp. For a tree (radial) network the hierarchy collapses and all three are equally tight. We now make this precise.

Consider their feasible sets $\mathbb{W}^{+}, \mathbb{W}_{c(G)}^{+}$and $\mathbb{W}_{G}^{+}$. We say that a set $A$ is an effective subset of a set $B$, denoted by $A \sqsubseteq B$, if, given a (partial) matrix $a \in A$, there is a (partial) matrix $b \in B$ that has the same cost $C(a)=C(b)$. We say $A$ is similar to $B$, denoted by $A \simeq B$, if $A \sqsubseteq B$ and $B \sqsubseteq A$. Note that $A \equiv B$ implies $A \simeq B$ but the converse may not be true. The feasible set of OPF (7) is an effective subset of the feasible sets of the relaxations; moreover these relaxations have similar feasible sets when the network is radial. This is a slightly different formulation of the same results in [57], [31].

Theorem 5: $\mathbb{V} \sqsubseteq \mathbb{W}^{+} \simeq \mathbb{W}_{c(G)}^{+} \sqsubseteq \mathbb{W}_{G}^{+}$. If $G$ is a tree then $\mathbb{V} \sqsubseteq \mathbb{W}^{+} \simeq \mathbb{W}_{c(G)}^{+} \simeq \mathbb{W}_{G}^{+}$.

Let $C^{\text {opt }}, C^{\text {sdp }}, C^{\text {ch }}, C^{\text {socp }}$ be the optimal values of OPF (7), OPF-sdp (21), OPF-ch (22), OPF-socp (23) respectively. Theorem 4 and Theorem 5 directly imply

Corollary 6: $C^{\mathrm{opt}} \geq C^{\mathrm{sdp}}=C^{\mathrm{ch}} \geq C^{\mathrm{socp}}$. If $G$ is a tree then $C^{\mathrm{opt}} \geq C^{\mathrm{sdp}}=C^{\mathrm{ch}}=C^{\mathrm{socp}}$.

Remark 7: Tightness. Theorem 5 and Corollary 6 imply that for radial networks one should always solve OPF-socp since it is the tightest and the simplest relaxation of the three. For mesh networks there is a tradeoff between OPF-socp and OPF-ch/OPF-sdp: the latter is tighter but requires heavier computation. Between OPF-ch and OPF-sdp, OPF-ch is usually preferable as they are equally tight but OPF-ch is usually much faster to solve for large sparse networks. See [28], [29], [31], [30], [66] for numerical studies that compare these relaxations. 


\section{F. Chordal relaxation}

Theorem 2 through Corollary 6 apply to any chordal extension $c(G)$ of $G$. The choice of $c(G)$ does not affect the optimal value of the chordal relaxation but determines its complexity. Unfortunately the optimal choice that minimizes the complexity of OPF-ch is NP-hard to compute.

This difficulty is due to two conflicting factors in choosing a $c(G)$. Recall that the constraint $W_{c(G)} \succeq 0$ in the definition of $\mathbb{W}_{c(G)}^{+}$consists of multiple constraints that the principal submatrices $W_{c(G)}(q) \succeq 0$, one for each (maximal) clique $q$ of $c(G)$. When two cliques $q$ and $q^{\prime}$ share a node their submatrices $W_{c(G)}(q)$ and $W_{c(G)}\left(q^{\prime}\right)$ share entries that must be decoupled by introducing auxiliary variables and equality constraints on these variables. The choice of $c(G)$ determines the number and sizes of these submatrices $W_{c(G)}(q)$ as well as the numbers of auxiliary variables and additional decoupling constraints. On the one hand if $c(G)$ contains few cliques $q$ then the submatrices $W_{c(G)}(q)$ tend to be large and expensive to compute (e.g. if $c(G)$ is the complete graph then there is a single clique, but $W_{c(G)}=W$ and OPF-ch is identical to OPF-sdp). On the other hand if $c(G)$ contains many small cliques $q$ then there tends to be more overlap and chordal relaxation tends to require more decoupling constraints. Hence choosing a good chordal extension $c(G)$ of $G$ is important but nontrivial. See [63], [64] and references therein for methods to compute efficient chordal relaxations of general QCQP. For OPF [30] proposes effective techniques to reduce the number of cliques in its chordal relaxation. To further reduce the problem size [66] proposes to carefully drop some of the decoupling constraints, though the resulting relaxation can be weaker.

\section{FEASIBLE SETS AND RELAXATIONS: BFM}

We now present an SOCP relaxation of OPF in BFM proposed in [23], [24] in two steps. We first relax the phase angles of $V$ and $I$ in (2) and then we relax a set of quadratic equalities to inequalities. This derivation pinpoints the difference between radial and mesh topologies. It motivates a recursive version of BFM for radial networks (Section VI) and the use of phase shifters for convexification of mesh networks (Part II [33]).

\section{A. Feasible sets}

Consider the following set of equations in the variables $x:=(S, \ell, v, s)$ in $\mathbb{R}^{3(m+n+1)}:^{4}$

$$
\begin{aligned}
\sum_{k: j \rightarrow k} S_{j k} & =\sum_{i: i \rightarrow j}\left(S_{i j}-z_{i j} \ell_{i j}\right)+s_{j}, \quad j \in N^{+} \\
v_{j}-v_{k} & =2 \operatorname{Re}\left(z_{j k}^{H} S_{j k}\right)-\left|z_{j k}\right|^{2} \ell_{j k}, \quad j \rightarrow k \in \tilde{E} \\
v_{j} \ell_{j k} & =\left|S_{j k}\right|^{2}, \quad j \rightarrow k \in \tilde{E}
\end{aligned}
$$

\footnotetext{
shorthand for

$$
\begin{aligned}
\sum_{k: j \rightarrow k} P_{j k} & =\sum_{i: i \rightarrow j}\left(P_{i j}-r_{i j} \ell_{i j}\right)+p_{j} \\
\sum_{k: j \rightarrow k} Q_{j k} & =\sum_{i: i \rightarrow j}\left(Q_{i j}-x_{i j} \ell_{i j}\right)+q_{j}
\end{aligned}
$$
}

${ }^{4}$ The use of complex variables is only a shorthand and should be interpreted as operations in real variables. For instance (24a) is a 
and define the solution set as:

$$
\mathbb{X}_{n c}:=\left\{x \in \mathbb{R}^{3(m+n+1)} \mid x \text { satisfies (3),(4),(24) }\right\}
$$

Note that the vector $v$ includes $v_{0}$ and $s$ includes $s_{0}$. The model (24) is first proposed in [25], [26]. ${ }^{5}$ It can be derived as a relaxation of BFM (2) as follows. Taking the squared magnitude of (2c) and replacing $\left|V_{j}\right|^{2}$ and $\left|I_{j k}\right|^{2}$ by $v_{j}$ and $\ell_{j k}$ respectively yield (24c). To obtain (24b), use (2b)-(2c) to write $V_{k}=V_{j}-z_{j k} S_{j k} V_{j}^{-1}$ and take the squared magnitude on both sides to eliminate the phase angles of $V$ and $I$. These operations define a mapping $h: \mathbb{C}^{2(m+n+1)} \rightarrow \mathbb{R}^{3(m+n+1)}$ by: for any $\tilde{x}=(S, I, V, s), h(\tilde{x}):=(S, \ell, v, s)$ with $\ell_{j k}=\left|I_{j k}\right|^{2}$ and $v_{j}=\left|V_{j}\right|^{2}$.

Throughout this paper we assume the cost function $C(\tilde{x})$ in OPF (9) depends on $\tilde{x}$ only through $x:=h(\tilde{x})$. For example for total real line loss $C(\tilde{x})=\sum_{(j, k) \in \tilde{E}} \operatorname{Re} z_{j k} \ell_{j k}$. If the cost is a weighted sum of real generation power then $C(\tilde{x})=\sum_{j}\left(c_{j} p_{j}+p_{j}^{d}\right)$ where $p_{j}$ are the real parts of $s_{j}$ and $p_{j}^{d}$ are the given real power demands at buses $j$; again $C(\tilde{x})$ depends only on $x$.

Then the model (24) is a relaxation of BFM (2) in the sense that the feasible set $\tilde{\mathbb{X}}$ of OPF in (9) is an effective subset of $\mathbb{X}_{n c}, \tilde{\mathbb{X}} \sqsubseteq \mathbb{X}_{n c}$, since $h(\tilde{\mathbb{X}}) \subseteq \mathbb{X}_{n c}$. We now characterize the subset of $\mathbb{X}_{n c}$ that is equivalent to $\tilde{\mathbb{X}}$.

Given an $x:=(S, \ell, v, s) \in \mathbb{R}^{3(m+n+1)}$ define $\beta(x) \in \mathbb{R}^{m}$ by

$$
\beta_{j k}(x):=\angle\left(v_{j}-z_{j k}^{H} S_{j k}\right), \quad j \rightarrow k \in \tilde{E}
$$

Even though $x$ does not include phase angles of $V, x$ implies a phase difference across each line $j \rightarrow k \in \tilde{E}$ given by $\beta_{j k}(x)$. The subset of $\mathbb{X}_{n c}$ that is equivalent to $\tilde{\mathbb{X}}$ are those $x$ for which there exists $\theta$ such that $\theta_{j}-\theta_{k}=\beta_{j k}(x)$. To state this precisely let $B$ be the $m \times n$ (transposed) reduced incidence matrix of $\tilde{G}$ :

$$
B_{l j}= \begin{cases}1 & \text { if edge } l \in \tilde{E} \text { leaves node } j \\ -1 & \text { if edge } l \in \tilde{E} \text { enters node } j \\ 0 & \text { otherwise }\end{cases}
$$

where $j \in N$. Consider the set of $x \in \mathbb{X}_{n c}$ such that

$$
\exists \theta \text { that solves } B \theta=\beta(x) \bmod 2 \pi
$$

i.e., $\beta(x)$ is in the range space of $B(\bmod 2 \pi)$. A solution $\theta(x)$, if exists, is unique in $(-\pi, \pi]^{n}$. Define the set

$$
\mathbb{X}:=\left\{x \in \mathbb{R}^{3(m+n+1)} \mid x \text { satisfies (3),(4),(24),(26) }\right\}
$$

The following result characterizes the feasible set $\tilde{\mathbb{X}}$ of OPF in BFM and follows from [24, Theorems 2 , 4].

Theorem 7: $\tilde{\mathbb{X}} \equiv \mathbb{X} \subseteq \mathbb{X}_{n c}$.

The bijection between $\tilde{\mathbb{X}}$ and $\mathbb{X}$ is given by $h$ defined above restricted to $\tilde{\mathbb{X}}$. Its inverse $h^{-1}(S, \ell, v, s)=$

\footnotetext{
${ }^{5}$ The original model, called the DistFlow equations, in [25], [26] is for radial (distribution) networks, but its extension here to mesh networks is trivial.
} 
$(S, I, V, s)$ is defined on $\mathbb{X}$ in terms of $\theta(x)$ by:

$$
\begin{aligned}
V_{j} & :=\sqrt{v_{j}} e^{\mathbf{i} \theta_{j}(x)}, \quad j \in N \\
I_{j k} & :=\sqrt{\ell_{j k}} e^{\mathbf{i}\left(\theta_{j}(x)-\angle S_{j k}\right)}, j \rightarrow k \in \tilde{E}
\end{aligned}
$$

The condition (26) is equivalent to the cycle condition (13) in the bus injection model. To see this fix any spanning tree $T=\left(N, E_{T}\right)$ of the (directed) graph $\tilde{G}$. We can assume without loss of generality (possibly after re-labeling the links) that $E_{T}$ consists of links $l=1, \ldots, n$. Then $B$ can be partitioned into

$$
B=\left[\begin{array}{l}
B_{T} \\
B_{\perp}
\end{array}\right]
$$

where the $n \times n$ submatrix $B_{T}$ corresponds to links in $T$ and the $(m-n) \times n$ submatrix $B_{\perp}$ corresponds to links in $T^{\perp}:=G \backslash T$. Similarly partition $\beta(x)$ into

$$
\beta(x)=\left[\begin{array}{l}
\beta_{T}(x) \\
\beta_{\perp}(x)
\end{array}\right]
$$

The next result, proved in [24, Theorems 2 and 4], provides a more explicit characterization of (26) in terms of $\beta(x)$. When it holds this characterization has the same interpretation of the cycle condition in (13): the voltage angle differences implied by $x$ sum to zero $(\bmod 2 \pi)$ around any cycle. Formally let $\tilde{\beta}$ be the extension of $\beta$ from directed to undirected links: for each $j \rightarrow k \in \tilde{E}$ let $\tilde{\beta}_{j k}(x):=\beta_{j k}(x)$ and $\tilde{\beta}_{k j}(x):=-\beta_{j k}(x)$. We say $c:=\left(j_{1}, \ldots, j_{K}\right)$ is an undirected cycle if, for each $k=1, \ldots, K$, either $j_{k} \rightarrow j_{k+1} \in \tilde{E}$ or $j_{k+1} \rightarrow j_{k} \in \tilde{E}$ with the interpretation that $j_{K+1}:=j_{1} ;\left(j_{k}, j_{k+1}\right) \in c$ denotes one of these links.

Theorem 8: An $x \in \mathbb{X}_{n c}$ satisfies (26) if and only if around each undirected cycle $c$ we have

$$
\sum_{(j, k) \in c} \tilde{\beta}_{j k}(x)=0 \quad \bmod 2 \pi
$$

In that case $\theta(x)=\mathscr{P}\left(B_{T}^{-1} \beta_{T}(x)\right)$ is the unique solution of $(26)$ in $(-\pi, \pi]^{n}$, where $\mathscr{P}(\phi)$ projects $\phi$ to $(-\pi, \pi]^{n}$.

Theorem 8 determines when the voltage magnitudes $v$ of a given $x$ can be assigned phase angles $\theta(x)$ so that the resulting $\tilde{x}:=h^{-1}(x)$ is a power flow solution in $\tilde{\mathbb{X}}$.

\section{B. SOCP relaxation}

The set $\mathbb{X}_{n c}$ that contains the (equivalent) feasible set $\mathbb{X}$ of OPF is still nonconvex because of the quadratic equalities in (24c). Relax them to inequalities:

$$
v_{j} \ell_{j k} \geq\left|S_{j k}\right|^{2}, \quad(j, k) \in \tilde{E}
$$

and define the set:

$$
\mathbb{X}^{+}:=\left\{x \in \mathbb{R}^{3(m+n+1)} \mid x\right. \text { satisfies (3),(4),(24a),(24b),(29)\} }
$$


Clearly $\tilde{\mathbb{X}} \stackrel{h}{\equiv} \mathbb{X} \subseteq \mathbb{X}_{n c} \subseteq \mathbb{X}^{+}$; see Figure 1 . Moreover $\mathbb{X}^{+}$is a second-order cone in the rotated form.

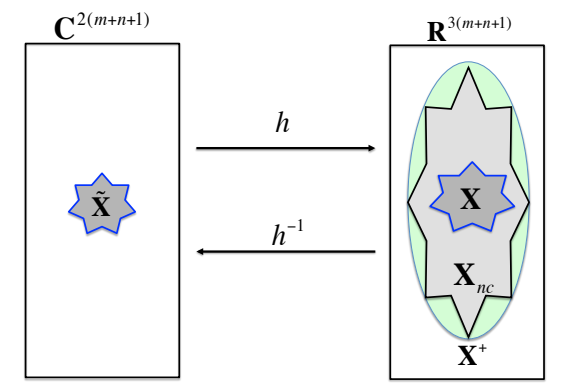

Fig. 1: Feasible sets $\tilde{\mathbb{X}}$ of OPF (9) in BFM, its equivalent set $\mathbb{X}$ (defined by $h$ ) and its relaxations $\mathbb{X}_{n c}$ and $\mathbb{X}^{+}$. If $\tilde{G}$ is a tree then $\mathbb{X}=\mathbb{X}_{n c}$.

The three sets $\mathbb{X}, \mathbb{X}_{n c}, \mathbb{X}^{+}$define the following problems:

\section{OPF:}

$$
\min _{x} C(x) \quad \text { subject to } \quad x \in \mathbb{X}
$$

\section{OPF-nc:}

$$
\min _{x} C(x) \quad \text { subject to } \quad x \in \mathbb{X}_{n c}
$$

\section{OPF-socp:}

$$
\min _{x} C(x) \quad \text { subject to } \quad x \in \mathbb{X}^{+}
$$

The next theorem follows from the results in [24] and implies that OPF (9) is equivalent to minimization over $\mathbb{X}$ and OPF-socp is its SOCP relaxation. Moreover for radial networks voltage and current angles can be ignored and OPF (9) is equivalent to OPF-nc.

Theorem 9: $\tilde{\mathbb{X}} \equiv \mathbb{X} \subseteq \mathbb{X}_{n c} \subseteq \mathbb{X}^{+}$. If $\tilde{G}$ is a tree then $\tilde{\mathbb{X}} \equiv \mathbb{X}=\mathbb{X}_{n c} \subseteq \mathbb{X}^{+}$.

Let $C^{\text {opt }}$ be the optimal cost of OPF (9) in the branch flow model. Let $C^{\text {opf }}, C^{\text {nc }}, C^{\text {socp }}$ be the optimal costs of OPF (30), OPF-nc (31), OPF-socp (32) respectively defined above. Theorem 9 implies

Corollary 10: $C^{\mathrm{opt}}=C^{\mathrm{opf}} \geq C^{\mathrm{nc}} \geq C^{\mathrm{socp}}$. If $\tilde{G}$ is a tree then $C^{\mathrm{opt}}=C^{\mathrm{opf}}=C^{\mathrm{nc}} \geq C^{\text {socp }}$.

Remark 8: SOCP relaxation. Suppose one solves OPF-socp and obtains an optimal solution $x^{\text {socp }}:=$ $(S, \ell, v, s) \in \mathbb{X}^{+}$. For radial networks if $x^{\text {socp }}$ attains equality in (29) then $x^{\text {socp }} \in \mathbb{X}_{n c}$ and Theorem 9 implies that an optimal solution $\tilde{x}^{\text {opt }}:=(S, I, V, s) \in \tilde{\mathbb{X}}$ of OPF (9) can be recovered from $x^{\text {socp }}$. Indeed $\tilde{x}^{\text {opt }}=$ $h^{-1}\left(x^{\text {socp }}\right)$ where $h^{-1}$ is defined in (27). Alternatively one can use the angle recovery algorithms in [24, Part I] to recover $\tilde{x}^{\text {opt }}$. For mesh networks $x^{\text {socp }}$ needs to both attain equality in (29) and satisfy the cycle condition (26) in order for an optimal solution $\tilde{x}^{\text {opt }}$ to be recoverable. Our experience with various practical test networks suggests that $x^{\text {socp }}$ usually attains equality in (29) but, for mesh networks, rarely satisfes (26) [23], [24], [56], [31]. Hence OPF-socp is effective for radial networks but not for mesh networks (in both BIM and BFM). 


\section{Equivalence}

Theorem 9 establishes a bijection between $\mathbb{X}$ and the feasible set $\tilde{\mathbb{X}}$ of OPF (9) in BFM. Theorem 4 establishes a bijection between $\mathbb{W}_{G}$ and the feasible set $\mathbb{V}$ of OPF (7) in BIM. Theorem 1 hence implies that $\mathbb{X} \equiv \tilde{\mathbb{X}} \equiv \mathbb{V} \equiv \mathbb{W}_{G}$. Moreover their SOCP relaxations are equivalent in these two models [57], [31]. Define the set of partial matrices defined on $G$ that are $2 \times 2$ psd rank-1 but do not satisfy the cycle condition (13):

$$
\mathbb{W}_{n c}:=\left\{W_{n c} \mid W_{G} \text { satisfies }(12), W_{G}(j, k) \succeq 0, \text { rank } W_{G}(j, k)=1 \text { for all }(j, k) \in E\right\}
$$

Clearly $\mathbb{W}_{G} \subseteq \mathbb{W}_{n c} \subseteq \mathbb{W}_{G}^{+}$in general and $\mathbb{W}_{G}=\mathbb{W}_{n c} \subseteq \mathbb{W}_{G}^{+}$for radial networks.

Theorem 11: $\mathbb{X} \equiv \mathbb{W}_{G}, \mathbb{X}_{n c} \equiv \mathbb{W}_{n c}$ and $\mathbb{X}^{+} \equiv \mathbb{W}_{G}^{+}$.

The bijection between $\mathbb{X}^{+}$and $\mathbb{W}_{G}^{+}$is a linear mapping defined as follows. Let $\mathbb{W}_{G} \subseteq \mathbb{C}^{2 m+n+1}$ denote the set of Hermitian partial matrices (including $\left[W_{G}\right]_{00}=v_{0}$ which is given). Let $x:=(S, \ell, v, s)$ denote vectors in $\mathbb{R}^{3(m+n+1)}$. Define the linear mapping $g: \mathbb{W}_{G}^{+} \rightarrow \mathbb{X}^{+}$by $x=g\left(W_{G}\right)$ where

$$
\begin{aligned}
S_{j k} & :=y_{j k}^{H}\left(\left[W_{G}\right]_{j j}-\left[W_{G}\right]_{j k}\right), \quad j \rightarrow k \\
\ell_{j k} & :=\left|y_{j k}\right|^{2}\left(\left[W_{G}\right]_{j j}+\left[W_{G}\right]_{k k}-\left[W_{G}\right]_{j k}-\left[W_{G}\right]_{k j}\right), j \rightarrow k \\
v_{j} & :=\left[W_{G}\right]_{j j}, \quad j \in N^{+} \\
s_{j} & :=\sum_{k: j \sim k} y_{j k}^{H}\left(\left[W_{G}\right]_{j j}-\left[W_{G}\right]_{j k}\right), \quad j \in N^{+}
\end{aligned}
$$

Its inverse $g^{-1}: \mathbb{X}^{+} \rightarrow \mathbb{W}_{G}^{+}$is $W_{G}=g^{-1}(x)$ where $\left[W_{G}\right]_{j j}:=v_{j}$ for $j \in N^{+}$and $\left[W_{G}\right]_{j k}:=v_{j}-z_{j k}^{H} S_{j k}=:\left[W_{G}\right]_{k j}^{H}$ for $j \rightarrow k$. The mapping $g$ (and its inverse $g^{-1}$ ) restricted to $\mathbb{W}_{G}\left(\mathbb{W}_{n c}\right)$ and $\mathbb{X}\left(\mathbb{X}_{n c}\right)$ define the bijection between them.

\section{BFM FOR RADIAL NETWORKS}

Theorem 9 implies that for radial networks the model (24) is exact. This is because the reduced incident matrix $B$ in (26) is $n \times n$ and invertible, so the cycle condition is always satisfied [24, Theorem 4]. Hence a solution in $\mathbb{X}_{n c}$ can be mapped to a branch flow solution in $\tilde{\mathbb{X}}$ by the mapping $h^{-1}$ defined in (27). For radial networks this model has two advantages: (i) it has a recursive structure that simplifies computation, and (ii) it has a linear approximation that provides simple bounds on branch powers $S_{j k}$ and voltage magnitudes $v_{j}$, as we now show.

\section{A. Recursive equations and graph orientation}

The model (24) holds for any graph orientation of $\tilde{G}$. It has a recursive structure when $\tilde{G}$ is a tree. In that case different orientations have different boundary conditions that initialize the recursion and may be convenient for different applications. Without loss of generality we take bus 0 as the root of the tree. We discuss two different orientations: one where every link points away from bus 0 and the other where every link points towards bus 0.6

\footnotetext{
${ }^{6} \mathrm{An}$ alternative model is to use an $u n$ directed graph and, for each link $(j, k)$, the variables $\left(S_{j k}, \ell_{j k}\right)$ and $\left(S_{k j}, \ell_{k j}\right)$ are defined for both directions, with the additional equations $S_{j k}+S_{k j}=z_{j k} \ell_{j k}$ and $\ell_{k j}=\ell_{j k}$.
} 
Case I: Links point away from bus 0 . Model (24) reduces to:

$$
\begin{aligned}
\sum_{k: j \rightarrow k} S_{j k} & =S_{i j}-z_{i j} \ell_{i j}+s_{j}, \quad j \in N^{+} \\
v_{j}-v_{k} & =2 \operatorname{Re}\left(z_{j k}^{H} S_{j k}\right)-\left|z_{j k}\right|^{2} \ell_{j k}, j \rightarrow k \in \tilde{E} \\
v_{j} \ell_{j k} & =\left|S_{j k}\right|^{2}, \quad j \rightarrow k \in \tilde{E}
\end{aligned}
$$

where bus $i$ in (33a) denotes the unique parent of node $j$ (on the unique path from node 0 to node $j$ ), with the understanding that if $j=0$ then $S_{i 0}:=0$ and $\ell_{i 0}:=0$. Similarly when $j$ is a leaf node ${ }^{7}$ all $S_{j k}=0$ in (33a). The model (33) is called the DistFlow equations and first proposed in [25], [26].

Its recursive structure is exploited in [48] to analyze the power flow solutions given an $\left(s_{j}, j \in N\right)$, as we now explain using the special case of a linear network with $n+1$ buses that represents a main feeder. To simplify notation denote $\left(S_{j(j+1)}, \ell_{j(j+1)}\right)$ and $z_{j(j+1)}$ by $\left(S_{j}, \ell_{j}\right)$ and $z_{j}$ respectively. Then the DistFlow equations (33) reduce to ( $v_{0}$ is given):

$$
\begin{aligned}
S_{j+1} & =S_{j}-z_{j} \ell_{j}+s_{j+1}, \quad j=0, \ldots, n-1 \\
v_{j+1} & =v_{j}-2 \operatorname{Re}\left(z_{j}^{H} S_{j}\right)+\left|z_{j}\right|^{2} \ell_{j}, \quad j=0, \ldots, n-1 \\
v_{j} \ell_{j} & =\left|S_{j}\right|^{2}, \quad j=0, \ldots, n-1 \\
S_{0} & =s_{0}, \quad S_{n}=0
\end{aligned}
$$

Let $x_{j}:=\left(S_{j}, \ell_{j}, v_{j}\right), j \in N^{+}$. If $s_{0}$ were known then one can start with $\left(v_{0}, s_{0}\right)$ and use the recursion (34a)(34c) to compute $x_{j}$ in terms of $s_{0}=S_{0}$, i.e., (34) can be collapsed into functions of the scalar variable $s_{0}$ (recall that $\left(s_{j}, j \in N\right)$ are given):

$$
x_{j}=f_{j}\left(s_{0}\right), \quad j \in N^{+}
$$

Use the boundary condition $(34 \mathrm{~d}), S_{n}=f_{n}\left(s_{0}\right)=0$, to solve for the scalar variable $s_{0}$. The other variables $x_{j}$ can then be computed from (35). This method can be extended to a general radial network with laterals [48]. See also [67], [68] for techniques for solving the nonlinear equations (35), and [54], [55] for a different recursive approach called the forward/backward sweep for radial networks.

Case II: Links point towards bus 0. Model (24) reduces to:

$$
\begin{aligned}
\hat{S}_{j i} & =\sum_{k: k \rightarrow j}\left(\hat{S}_{k j}-z_{k j} \hat{\ell}_{k j}\right)+s_{j}, \quad j \in N^{+} \\
\hat{v}_{k}-\hat{v}_{j} & =2 \operatorname{Re}\left(z_{k j}^{H} \hat{S}_{k j}\right)-\left|z_{k j}\right|^{2} \hat{\ell}_{k j}, \quad k \rightarrow j \in \tilde{E} \\
\hat{v}_{k} \hat{\ell}_{k j} & =\left|\hat{S}_{k j}\right|^{2}, \quad k \rightarrow j \in \tilde{E}
\end{aligned}
$$

where $i$ in (36a) denotes the node on the unique path between node 0 and node $j$. The boundary condition is defined by $S_{j i}=0$ in (36a) when $j=0$ and $S_{k j}=0, \ell_{k j}=0$ in (36a) when $j$ is a leaf node. An advantage of this orientation is illustrated in the next subsection in proving a simple bound on $\hat{v}_{j}$. The proof establishes

\footnotetext{
${ }^{7} \mathrm{~A}$ node $j$ is a leaf node if there exists no $i$ such that $i \rightarrow j \in \tilde{E}$.
} 
formally that there is a bijection between the solution set of (33) and that of (36):

$$
\begin{aligned}
-S_{k j} & \leftrightarrow \hat{S}_{j k}-z_{j k} \hat{\ell}_{j k} \\
\ell_{k j} & \leftrightarrow \hat{\ell}_{j k} \\
v_{j} & \leftrightarrow \hat{v}_{j}
\end{aligned}
$$

\section{B. Linear approximation and bounds}

By setting $\ell_{j k}=0$ in (33) we obtain a linear approximation of the the branch flow model, with the graph orientation where all links point away from bus 0 :

$$
\begin{aligned}
\sum_{k: j \rightarrow k} S_{j k}^{\operatorname{lin}} & =S_{i j}^{\operatorname{lin}}+s_{j}, \quad j \in N^{+} \\
v_{j}^{\operatorname{lin}}-v_{k}^{\operatorname{lin}} & =2 \operatorname{Re}\left(z_{j k}^{H} S_{j k}^{\operatorname{lin}}\right), \quad j \rightarrow k \in E
\end{aligned}
$$

where bus $i$ in (37a) denotes the unique parent of bus $j$. The boundary condition is: $S_{i 0}^{\operatorname{lin}}:=0$ in (37a) when $j=0$, and $S_{j k}^{\text {lin }}=0$ in (37a) when $j$ is a leaf node. This is called the simplified DistFlow equations in [26], [69]. It is a good approximation of (33) because the loss $z_{j k} \ell_{j k}$ is typically much smaller than the branch power flow $S_{j k}$.

The next result provides simple bounds on $(S, v)$ in terms of their linear approximations $\left(S^{\operatorname{lin}}, v^{\text {lin }}\right)$. Denote by $\mathbb{T}_{j}$ the subtree rooted at bus $j$, including $j$. We write " $k \in \mathbb{T}_{j}$ " to mean node $k$ of $\mathbb{T}_{j}$ and " $(k, l) \in \mathbb{T}_{j}$ " to mean edge $(k, l)$ of $\mathbb{T}_{j}$. Denote by $\mathbb{P}_{k}$ the set of links on the unique path from bus 0 to bus $k$.

Lemma 12: Fix any $v_{0}$ and $s \in \mathbb{R}^{2(n+1)}$. Let $(S, \ell, v)$ and $\left(S^{\operatorname{lin}}, v^{\text {lin }}\right)$ be solutions of (33) and (37) respectively with the given $v_{0}$ and $s$. Then

(1) For $i \rightarrow j \in E$

$$
\begin{aligned}
S_{i j}^{\operatorname{lin}} & =-\sum_{k \in \mathbb{T}_{j}} s_{k} \\
S_{i j} & =-\sum_{k \in \mathbb{T}_{j}} s_{k}+\left(z_{i j} \ell_{i j}+\sum_{(k, l) \in \mathbb{T}_{j}} z_{k l} \ell_{k l}\right)
\end{aligned}
$$

(2) For $i \rightarrow j \in E, S_{i j} \geq S_{i j}^{\text {lin }}$ with equality if only if $\ell_{i j}$ and all $\ell_{k l}$ in $\mathbb{T}_{j}$ are zero.

(3) For $j \in N^{+}$

$$
\begin{aligned}
v_{j}^{\operatorname{lin}} & =v_{0}-\sum_{(i, k) \in \mathbb{P}_{j}} 2 \operatorname{Re}\left(z_{i k}^{H} S_{i k}^{\text {lin }}\right) \\
v_{j} & =v_{0}-\sum_{(i, k) \in \mathbb{P}_{j}}\left(2 \operatorname{Re}\left(z_{i k}^{H} S_{i k}\right)-\left|z_{i k}\right|^{2} \ell_{i k}\right)
\end{aligned}
$$

(4) For $j \in N^{+}, v_{j} \leq v_{j}^{\operatorname{lin}}$.

Lemma 12 says that the power flow $S_{i j}$ on line $(i, j)$ equals the total load $-\sum_{k \in \mathbb{T}_{j}} s_{k}$ in the subtree rooted at node $j$ plus the total line loss in supplying these loads. The linear approximation $S_{i j}^{\text {lin }}$ neglects the line losses and underestimates the required power to supply these loads. 
Lemma 12(1)-(3) can be easily proved by recursing on (33a)-(33b) and (37). Since $S_{i j} \geq S_{i j}^{\text {lin but }}\left|z_{i k}\right|^{2} \ell_{i k} \geq$ 0 , a direct proof of Lemma 12(4) is not obvious. Instead, one can make use of Lemma 13 below and define a bijection between the solutions $(S, \ell, v)$ of (33) and the solutions $(\hat{S}, \hat{\ell}, \hat{v})$ of (36) in which $v=\hat{v}$. It can be checked that the solutions of (37) and those of (38) are related by $S^{\text {lin }}=-\hat{S}^{\text {lin }}$ and $v^{\text {lin }}=\hat{v}^{\text {lin }}$. Then Lemma 13(4) implies Lemma 12(4).

A linear approximation of (36) is (setting $\left.\hat{\ell}_{k j}=0\right)$ :

$$
\begin{aligned}
\hat{S}_{j i}^{\operatorname{lin}} & =\sum_{k: k \rightarrow j} \hat{S}_{k j}^{\mathrm{lin}}+s_{j}, \quad j \in N^{+} \\
\hat{v}_{k}^{\mathrm{lin}}-\hat{v}_{j}^{\mathrm{lin}} & =2 \operatorname{Re}\left(z_{k j}^{H} \hat{S}_{k j}^{\mathrm{lin}}\right), k \rightarrow j \in \tilde{E}
\end{aligned}
$$

Lemma 13: Fix any $v_{0}$ and $s \in \mathbb{R}^{2(n+1)}$. Let $(\hat{S}, \hat{\ell}, \hat{v})$ and $\left(\hat{S}^{\mathrm{lin}}, \hat{v}^{\text {lin }}\right)$ be solutions of (36) and (38) respectively with the given $v_{0}$ and $s$. Then

(1) For all $j \rightarrow i \in E$

$$
\begin{aligned}
\hat{S}_{j i}^{\operatorname{lin}} & =\sum_{k \in \mathbb{T}_{j}} s_{k} \\
\hat{S}_{j i} & =\sum_{k \in \mathbb{T}_{j}} s_{k}-\sum_{(k, l) \in \mathbb{T}_{j}} z_{k l} \hat{\ell}_{k l}
\end{aligned}
$$

(2) For all $j \rightarrow i \in E, \hat{S}_{j i} \leq \hat{S}_{j i}^{\text {lin }}$ with equality if and only if all $\ell_{k l}$ in $\mathbb{T}_{j}$ are zero.

(3) For $j \in N^{+}$

$$
\begin{aligned}
\hat{v}_{j}^{\text {lin }} & =v_{0}+\sum_{(i, k) \in \mathbb{P}_{j}} 2 \operatorname{Re}\left(z_{i k}^{H} \hat{S}_{i k}^{\text {lin }}\right) \\
\hat{v}_{j} & =v_{0}+\sum_{(i, k) \in \mathbb{P}_{j}}\left(2 \operatorname{Re}\left(z_{i k}^{H} \hat{S}_{i k}\right)-\left|z_{i k}\right|^{2} \hat{\ell}_{i k}\right)
\end{aligned}
$$

(4) For $j \in N^{+}, \hat{v}_{j} \leq \hat{v}_{j}^{\text {lin }}$.

Lemma 13 says that the branch power $\hat{S}_{j i}$ (towards bus 0) equals the total power injection $\sum_{k \in \mathbb{T}_{j}} s_{k}$ in the subtree rooted at bus $j$ minus the line losses in that subtree. The linear approximation $\hat{S}_{j i}^{\mathrm{lin}}$ neglects the line losses and hence overestimates the branch power flow. Lemma 13 can be easily proved by recursing on (36a)-(36b) and (38).

Remark 9: Bounds for SOCP relaxation. Lemmas 12 and 13 do not depend on the quadratic equalities (33c) and (36c) as long as $\ell_{j k} \geq 0$. In particular the lemmas hold if the equalities have been relaxed to inequalities $v_{j} \ell_{j k} \geq\left|S_{j k}\right|^{2}$. These bounds are used in [56] to prove a sufficient condition for exact SOCP relaxation for radial networks.

Remark 10: Linear approximations. For radial networks the linear approximations (37) and (38) of BFM have two advantages over the (linear) DC approximation of BIM. First they have a simple recursive structure that leads to simple bounds on power flow quantities. Second DC approximation assumes $r_{j k}=0$, fixes voltage magnitudes, and ignores reactive power, whereas (37) and (38) do not. This is important for distribution systems where $r_{j k}$ are not negligible, voltages can fluctuate significantly and reactive powers are used to regulate them. On the other hand (37) and (38) are applicable only for radial networks whereas 
DC approximation applies to mesh networks as well. See also [20] for a more accurate linearization of BIM that addresses the shortcomings of DC OPF.

\section{CONCLUSION}

We have presented a bus injection model and a branch flow model, formulated several relaxations of OPF, and proved their relations. These results suggest a new approach to solving OPF summarized in Figure 2. For radial networks we recommend solving OPF-socp in either BIM or BFM though there is preliminary

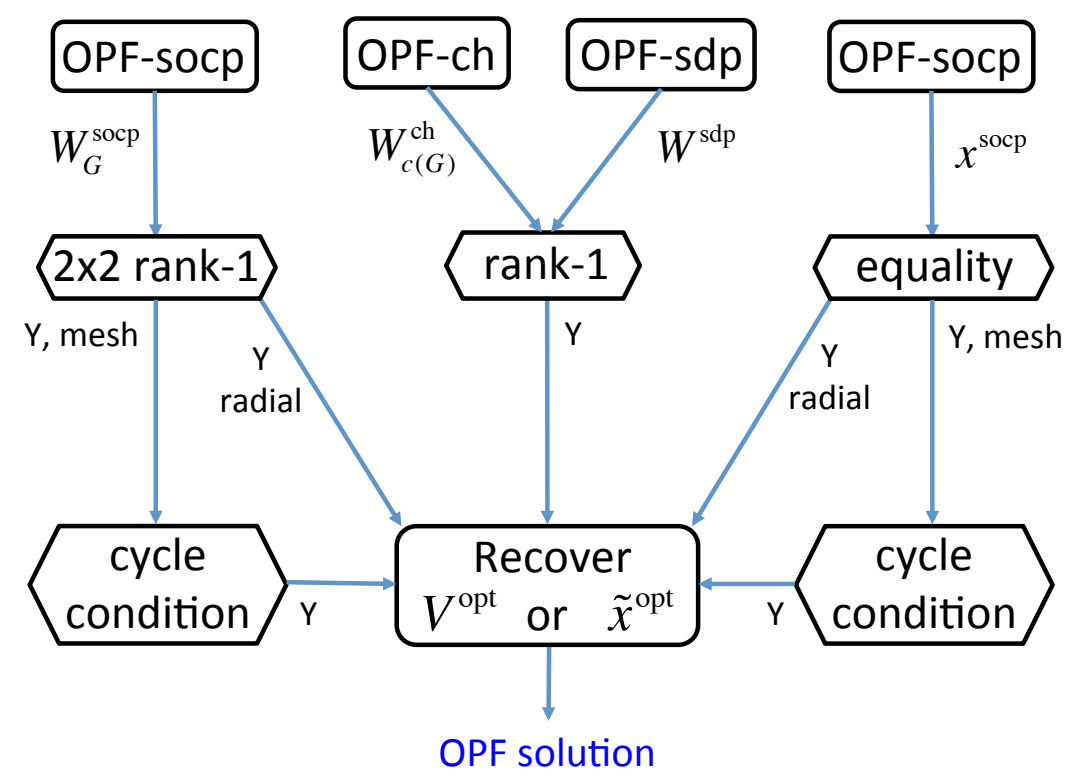

Fig. 2: Solving OPF through semidefinite relaxations.

evidence that BFM can be more stable numerically. For mesh networks we recommend solving OPF-ch for small networks and OPF-socp followed by a heuristic search for a feasible point for large networks. Also see Remarks 7 and 8.

The key for this solution strategy is that the relaxations are exact so that an optimal solution of the original OPF can be recovered. In Part II of this paper [33] we summarize sufficient conditions that guarantee exact relaxation. 


\section{APPENDIX}

\section{VIII: MATHEMATICAL PRELIMINARIES}

In this appendix we summarize some basic concepts in optimization, matrix completion and chordal relaxation that we use in this two-part tutorial. For notations see Section I. More details can be found in, e.g., [70], [71], [72], [63], [64], [73], [74], [62].

\section{A. QCQP, SDP, SOCP}

Quadratic constrained quadratic program (QCQP) is the following problem:

$$
\begin{aligned}
\min _{x \in \mathbb{C}^{n}} & x^{H} C_{0} x \\
\text { subject to } & x^{H} C_{l} x \leq b_{l}, \quad l=1, \ldots, L
\end{aligned}
$$

where $x \in \mathbb{C}^{n}$, for $l=0, \ldots, L, C_{l} \in \mathbb{S}^{n}$ (so that $x^{H} C_{l} x$ are real), and $b_{l} \in \mathbb{R}$ are given. If $C_{l}, l=0, \ldots, L$, are positive semidefinite then (39) is a convex QCQP. Otherwise it is generally nonconvex.

Any psd rank-1 matrix $X$ has a unique spectral decomposition $X=x x^{H}$. Using $x^{H} C_{l} x=\operatorname{tr} C_{l} x x^{H}=: \operatorname{tr} C_{l} X$ we can rewrite a QCQP as the following equivalent problem where the optimization is over Hermitian matrices:

$$
\begin{aligned}
\min _{X \in \mathbb{S}^{n}} & \operatorname{tr} C_{0} X \\
\text { subject to } & \operatorname{tr} C_{l} X \leq b_{l}, \quad l=1, \ldots, L \\
& X \succeq 0, \quad \operatorname{rank} X=1
\end{aligned}
$$

While the objective function and the constraints in (39) is quadratic in $x$ they are linear in $X$ in (40a)-(40b). The constraint $X \succeq 0$ in (40c) is convex ( $\mathbb{S}_{+}^{n}$ is a convex cone). The rank constraint in (40c) is the only nonconvex constraint. Removing the rank constraint results in a semidefinite program (SDP):

$$
\begin{aligned}
\min _{X \in \mathbb{S}^{n}} & \operatorname{tr} C_{0} X \\
\text { subject to } & \operatorname{tr} C_{l} X \leq b_{l}, \quad l=1, \ldots, L \\
& X \succeq 0
\end{aligned}
$$

SDP is a convex program and can be efficiently computed. We call (41) an SDP relaxation of QCQP (39) because the feasible set of (40) is a subset of the feasible set of SDP (41). A strategy for solving QCQP (39) is to solve SDP (41) for an optimal $X^{\text {opt }}$ and check its rank. If rank $X^{\text {opt }}=1$ then $X^{\text {opt }}$ is optimal for (40) as well and an optimal solution $x^{\text {opt }}$ of QCQP (39) can be recovered from $X^{\text {opt }}$ through spectral decomposition $X^{\text {opt }}=x^{\text {opt }}\left(x^{\text {opt }}\right)^{H}$. If rank $X^{\text {opt }}>1$ then, in general, no feasible solution of QCQP can be directly obtained from $X^{\text {opt }}$ but the optimal objective value of SDP provides a lower bound on that of QCQP.

To derive the Lagrangian dual of SDP (41), form the Lagrangian, for $y:=\left(y_{l}, l=1, \ldots, L\right) \geq 0$,

$$
L(X ; y):=\operatorname{tr} C_{0} X+\sum_{l=1}^{L} y_{l}\left(\operatorname{tr} C_{l} X-b_{l}\right)=\operatorname{tr}\left(C_{0}+\sum_{l} y_{l} C_{l}\right) X-b^{T} y
$$


Then the primal problem (41) is equivalent to $\min _{X \succeq 0} \max _{y \geq 0} L(X ; y)$ and its dual is $\max _{y \geq 0} \min _{X \succeq 0} L(X ; y)$ (if we allow their objective values to be $\pm \infty$ ). Hence the dual objective function is

$$
\min _{X \succeq 0} L(X ; y)= \begin{cases}-b^{T} y & \text { if } C_{0}+\sum_{l} y_{l} C_{l} \preceq 0 \\ -\infty & \text { otherwise }\end{cases}
$$

Hence the dual problem is:

$$
\min _{y \geq 0} b^{T} y \quad \text { subject to } \quad C_{0}+\sum_{l=1}^{L} y_{l} C_{l} \preceq 0
$$

A pair $\left(X^{\mathrm{opt}}, y^{\mathrm{opt}}\right)$ is a primal-dual optimal if and only if

1) Primal feasibility: $X^{\mathrm{opt}} \succeq 0$ and $\operatorname{tr} C_{l} X^{\mathrm{opt}} \leq b_{l}, l=1, \ldots, L$.

2) Dual feasibility: $y^{\mathrm{opt}} \geq 0$ and $C_{0}+\sum_{l=1}^{L} y_{l}^{\mathrm{opt}} C_{l} \preceq 0$.

3) Complementary slackness: $\operatorname{tr}\left(C_{0}+\sum_{l} y_{l}^{\mathrm{opt}} C_{l}\right) X^{\mathrm{opt}}=0$.

A special case of SDP is a second-order cone program (SOCP):

$$
\begin{aligned}
\min _{x \in \mathbb{C}^{n}} & c_{0}^{H} x \\
\text { subject to } & \left\|C_{l} x+b_{l}\right\| \leq c_{l}^{H} x+d_{l}, \quad l=1, \ldots, L
\end{aligned}
$$

where $c_{0} \in \mathbb{C}^{n}$ defines the cost and, for $l=1, \ldots, L, C_{l} \in \mathbb{C}^{\left(n_{l}-1\right) \times n}, b_{l} \in \mathbb{C}^{n_{l}-1}, c_{l} \in \mathbb{C}^{n}$, and $d_{l} \in \mathbb{R}$ are given. Here $c_{l}, l=0, \ldots, L$, are such that $c_{l}^{H} x$ are real and $\|\cdot\|$ is the Euclidean norm, $\|u\|:=\sqrt{u^{H} u}$. The feasible set defined by (42b) is called a second-order cone and is a convex set. SOCP includes linear program and convex QCQP as special cases [72]. Even though an SOCP can be formulated as a standard SDP, solving an SOCP via SDP is generally much less efficient. The number of iterations to reduce the duality gap to a constant fraction of itself is bounded above by $O(\sqrt{L})$ for SOCP and by $O\left(\sqrt{\sum_{l} n_{l}}\right)$ for SDP [72]. Moreover each iteration is much faster for SCOP than for SDP.

For optimal power flow problems, we use SOCP in the following rotated form:

$$
\begin{aligned}
\min _{x \in \mathbb{C}^{n}} & c_{0}^{H} x \\
\text { subject to } & \left\|C_{l} x+b_{l}\right\|^{2} \leq\left(c_{l}^{H} x+d_{l}\right)\left(\hat{c}_{l}^{H} x+\hat{d}_{l}\right), \quad l=1, \ldots, L
\end{aligned}
$$

This can be converted to the standard form (42) via the transformation: for any complex vector $u \in \mathbb{C}^{l}$, any real numbers $a, b \in \mathbb{R}$,

$$
\|u\|^{2} \leq a b, a \geq 0, b \geq 0 \Leftrightarrow\left\|\left[\begin{array}{c}
2 u \\
a-b
\end{array}\right]\right\| \leq a+b
$$

In this paper we formulate optimal power flow (OPF) problems as QCQPs and describe SDP and SOCP relaxations of OPF. The third relaxation we will discuss is chordal relaxation based on the notion of chordal extension of a network graph. We now review some basic concepts in graph theory, partial matrices and completions, and show that a chordal relaxation is indeed a semidefinite program. 


\section{B. Graph, partial matrix and completion}

Consider a graph $G=(N, E)$ with $N:=\{1, \ldots, n\} . G$ can either be undirected or directed with an arbitrary orientation. Two nodes $j$ and $k$ are adjacent if $j \sim k \in E$. A complete graph is one where every pair of nodes is adjacent. A subgraph of $G$ is a graph $F=\left(N^{\prime}, E^{\prime}\right)$ with $N^{\prime} \subseteq N$ and $E^{\prime} \subseteq E$. A clique of $G$ is a complete subgraph of $G$. A maximal clique of $G$ is a clique that is not a subgraph of another clique of $G$.

By a path connecting nodes $j$ and $k$ we mean either a set of distinct nodes $\left(j, n_{1}, \ldots, n_{i}, k\right)$ such that $\left(j \sim n_{1}\right),\left(n_{1} \sim n_{2}\right), \ldots,\left(n_{i} \sim k\right)$ are edges in $E$ or this set of edges, depending on the context. A cycle $\left(n_{1}, \ldots, n_{i}\right)$ is a path such that $\left(n_{1} \sim n_{2}\right), \ldots,\left(n_{i} \sim n_{1}\right)$ are edges in $E$. By convention we exclude a pair of adjacent nodes $(j, k)$ as a cycle. We will only consider connected graphs in which there is a path between every pair of nodes.

A cycle in $G$ that has no chord (an edge connecting two nodes that are non-adjacent in the cycle) is called a minimal cycle. $G$ is chordal if all its minimal cycles are of length 3 (recall that an edge $(j, k)$ is not considered a cycle). A chordal extension of $G$ is a chordal graph on the same set of nodes as $G$ that contains $G$ as a subgraph. Every graph has a chordal extension; e.g. the complete graph on the same set of nodes is a trivial chordal extension.

Fix a graph $G=(N, E)$ with $N:=\{1, \ldots, n\}$ and $E \subseteq N \times N$. For our purposes here we assume $G$ is undirected so that $(j, k) \in E$ if and only if $(k, j) \in E$. A $G$-partial matrix (or simply a partial matrix if $G$ is clear from the context) is a set of complex numbers:

$$
X_{G}:=\left(\left[X_{G}\right]_{j j} \in \mathbb{C}, j \in N,\left[X_{G}\right]_{j k} \in \mathbb{C},(j, k) \in E\right)
$$

One can treat a partial matrix $X_{G}$ as entries of an $n \times n$ matrix $X$ whose entries $X_{j k}$ are unspecified if $(j, k) \notin E$. See Figure 3(a) below for an example. Given a partial matrix $X_{G}$ we call an $n \times n$ matrix $X$ a completion of $X_{G}$ if $X_{j j}=\left[X_{G}\right]_{j j}, j \in N$, and $X_{j k}=\left[X_{G}\right]_{j k},(j, k) \in E$, i.e., $X$ agrees with $X_{G}$ on $G{ }^{8}$

Consider any $n \times n$ matrix $X$. Given any $k \leq n$ nodes $\left(n_{1}, n_{2}, \ldots, n_{k}\right)$ let $X\left(n_{1}, \ldots, n_{k}\right)$ denote the $k \times k$ principal submatrix of $X$ defined by:

$$
\left[X\left(n_{1}, \ldots, n_{k}\right)\right]_{i j}:=X_{i j}, \quad i, j \in\left\{n_{1}, \ldots, n_{k}\right\}
$$

Any maximal clique $q:=\left(n_{1}, n_{2}, \ldots, n_{k}\right)$ of $G$ with $k$ nodes defines a (fully specified) $k \times k$ principal submatrix denoted by $X(q):=X\left(n_{1}, \ldots, n_{k}\right)$. In particular each edge $(i, j) \in E$ is a clique and defines a $2 \times 2$ principal submatrix $X(i, j)$, which we use heavily in discussing optimal power flow problems. These notions are extended to partial matrices with $X$ replaced by $X_{G}$.

We extend the notions of Hermitian, psd, rank-1, and trace to partial matrices as follows. We say that a partial matrix $X_{G}$ is Hermitian, denoted by $X_{G}=X_{G}^{H}$, if $\left[X_{G}\right]_{k j}=\left(\left[X_{G}\right]_{j k}\right)^{H}$. An $n \times n$ matrix $X$ is psd if and only if all its principal submatrices (including $X$ itself) is psd. We extend the notion of psd to partial matrices using this property, by saying that a partial matrix $X_{G}$ is psd if all its "principal submatrices" that are fully specified are psd. Formally $X_{G}$ is psd, denoted by $X_{G} \succeq 0$, if $X_{G}(q) \succeq 0$ for all maximal cliques $q$ of $G$. Note that if $X_{G}$ is psd then it is Hermitian by definition. Similarly we say that a partial matrix $X_{G}$

\footnotetext{
${ }^{8}$ We abuse the $X_{G}$ notation: given $G, X_{G}$ is a partial matrix defined on $G$, and given an $n \times n$ matrix $X, X_{G}$ is the submatrix $\left(X_{j j}, j \in\right.$ $\left.N, X_{j k},(j, k) \in E\right)$ of $X$ defined by $G$. The meaning should be clear from the context.
} 
is rank-1, denoted by rank $X_{G}=1$, if $X_{G}(q)$ is rank-1 for all maximal cliques $q$ of $G$. We say $W_{G}$ is $2 \times 2$ psd on $G$ if, for all $(j, k) \in E$, the $2 \times 2$ matrices $W_{G}(j, k)$ are psd, i.e.,

$$
\left[W_{G}\right]_{j j} \geq 0, \quad\left[W_{G}\right]_{k k} \geq 0, \quad\left[W_{G}\right]_{j j}\left[W_{G}\right]_{k k} \geq\left|\left[W_{G}\right]_{j k}\right|^{2}
$$

We say $W_{G}$ is $2 \times 2$ rank- 1 on $G$ if, for all $(j, k) \in E, W_{G}(j, k)$ are $2 \times 2$ rank-1 matrices, i.e., they are not the zero matrices and

$$
\left[W_{G}\right]_{j j}\left[W_{G}\right]_{k k}=\left|\left[W_{G}\right]_{j k}\right|^{2}
$$

Finally we say that an $n \times n$ matrix $C$ is defined on graph $G$ if $C_{j k}=0$ if $(j, k) \notin E$. We extend the operation tr to partial matrices $X_{G}$ : if $C$ and $X_{G}$ are defined on the same graph $G$ then

$$
\operatorname{tr} C X_{G}=\sum_{j \in N} C_{j j}\left[X_{G}\right]_{j j}+\sum_{(j, k) \in E} C_{j k}\left[X_{G}\right]_{j k}
$$

Suppose the matrices $C_{l}$ in (41), $l=0, \ldots, L$, are all defined on $G$, i.e., for all $l,\left[C_{l}\right]_{j k}=0$ if $(j, k) \notin E$. Then given any $n \times n$ matrix $X, \operatorname{tr} C_{l} X=\operatorname{tr} C_{l} X_{G}$ where $X_{G}$ is the submatrix of $X$ defined by $G$. Conversely, given a partial matrix $X_{G}$ that satisfies (41b), any completion $X$ of $X_{G}$ satisfies (41b). Even though both the objective function (41a) and the constraints (41b) depend only on the partial matrix $X_{G}$, the constraint $X \succeq 0$ in (41c) depends also on entries not in $X_{G}$. Indeed the number of complex variables in $X$ is $n^{2}$ while the number of complex variables in $X_{G}$ is only $n+2|E|$, which is much smaller than $n^{2}$ if $G$ is large but sparse. Hence instead of solving for a full psd matrix $X$ directly as in SDP (41) we would like to compute a partial matrix $X_{G}$ that has a psd completion $X$ that satisfies (41b)-(41c). If the completion $X$ is rank-1 then it also solves the problem (40) and hence yields a solution to the original QCQP (39) through spectral decomposition of $X$. Theorem 2 provides an exact characterization of when this is possible.

To solve the QCQP (39), Theorem 2 suggests the following strategy that exploits the sparsity of graph $G$ : instead of solving SDP (41) for a psd matrix $X^{\text {opt }} \in \mathbb{S}_{+}^{n}$, solve for a psd partial matrix $X_{F}^{\text {opt }}$ defined on a chordal extension $F$ of $G$. If the solution $X_{F}^{\text {opt }}$ turns out to be rank-1 as well then an optimal solution $x^{\text {opt }}$ of QCQP (39) can be recovered from $X_{F}^{\text {opt }}$ (see Section IV-D).

Two questions naturally arise in this approach: (i) How to formulate a semidefinite relaxation based on a given a chordal extension $F$ of $G$ ? (ii) How to choose a good chordal extension $F$ of $G$ so that the resulting relaxation can be solved efficiently? We next illustrate the issues involved in these two questions through an example. See [63], [64] for more details.

\section{Chordal relaxation}

Fix a graph $G=(N, E)$. Let $F=\left(N, E^{\prime}\right)$ be a chordal extension of $G$ with $E^{\prime} \supseteq E$. Let $q_{1}, \ldots, q_{K}$ be the set of maximal cliques of $F$ and $X\left(q_{k}\right), k=1, \ldots, K$, be the set of principal submatrices of $X$ defined on these cliques. Consider the following problem where the optimization variable is the Hermitian partial 
matrix $W_{F} \in \mathbb{C}^{n+2\left|E^{\prime}\right|}$ defined on the chordal extension $F$ :

$$
\begin{aligned}
\min _{X_{F}=X_{F}^{H}} & \operatorname{tr} C_{0} X_{G} \\
\text { subject to } & \operatorname{tr} C_{l} X_{G} \leq b_{l}, \quad l=1, \ldots, L \\
& X_{F}\left(q_{k}\right) \succeq 0, \quad k=1, \ldots, K
\end{aligned}
$$

We call this problem a chordal relaxation of QCQP (39). Recall that we assume $C_{l}, l=0, \ldots, L$, are all defined on $G$, i.e., $\left[C_{l}\right]_{j k}=0$ if $(j, k) \notin E$. This implies that $\operatorname{tr} C_{l} X=\operatorname{tr} C_{l} X_{F}=\operatorname{tr} C_{l} X_{G}$. Then chordal relaxation (43) is equivalent to SDP (41) in the sense that given any feasible solution $X_{F}$ of (43), there is a psd completion $X$ that is feasible for (41) and has the same cost, and vice versa. This is a consequence of [62, Theorem 7] that says every psd partial matrix has a psd completion if and only if the underlying graph is chordal. See also Theorem 5 and Corollary 6.

The first step in constructing the chordal relaxation (43) is to list all the maximal cliques $q_{k}$. Even though listing all maximal cliques of a general graph is NP-hard it can be done efficiently for a chordal graph. This is because a graph is chordal if and only if it has a perfect elimination ordering [75] and computing this ordering takes linear time in the number of nodes and edges [76]. Given a perfect elimination ordering all maximal cliques $q_{k}$ can be enumerated and $X_{F}\left(q_{k}\right)$ constructed efficiently [63]. For optimal power flow problems the computation depends only on the topology of the power network, not on operational data, and therefore can be done offline.

We now show that (43) is indeed an SDP by converting it into the standard form (41) with the introduction of auxiliary variables, following the procedure described in [63]. This conversion also illustrates the difficulty in choosing a good chordal extension $F$ (see Remark 11 below).

The (fully specified) matrices $X_{F}\left(q_{k}\right)$ in (43c) can be treated as principal submatrices of an $n \times n$ matrix $X$. They may not however be integrated directly into a common $n \times n$ matrix variable $X$ because different $X_{F}\left(q_{k}\right)$ may share entries. We now explain the issue and its resolution using the example in Figure 1. They are the same in the general case with more cumbersome notations; see [63], [64]. Suppose we have chosen the chordal extension $F$ in Figure 3(b) with two overlapping cliques $q_{1}$ and $q_{2}$ as explained in the caption of the figure. To decouple the two matrices $X_{F}\left(q_{1}\right)$ and $X_{F}\left(q_{2}\right)$, define the $3 \times 3$ matrix

$$
X^{\prime}\left(q_{1}\right):=\left[\begin{array}{lll}
x_{11} & x_{12} & x_{13} \\
x_{21} & u_{22} & u_{23} \\
x_{31} & u_{32} & u_{33}
\end{array}\right]
$$

where the decoupling variables $u_{j k}$ are constrained to be:

$$
u_{j k}=x_{j k} \text { for } j, k=2,3
$$

The constraints (43c) are replaced by

$$
X_{F}^{\prime}\left(q_{1}\right) \succeq 0 \quad \text { and } \quad X_{F}\left(q_{2}\right) \succeq 0
$$



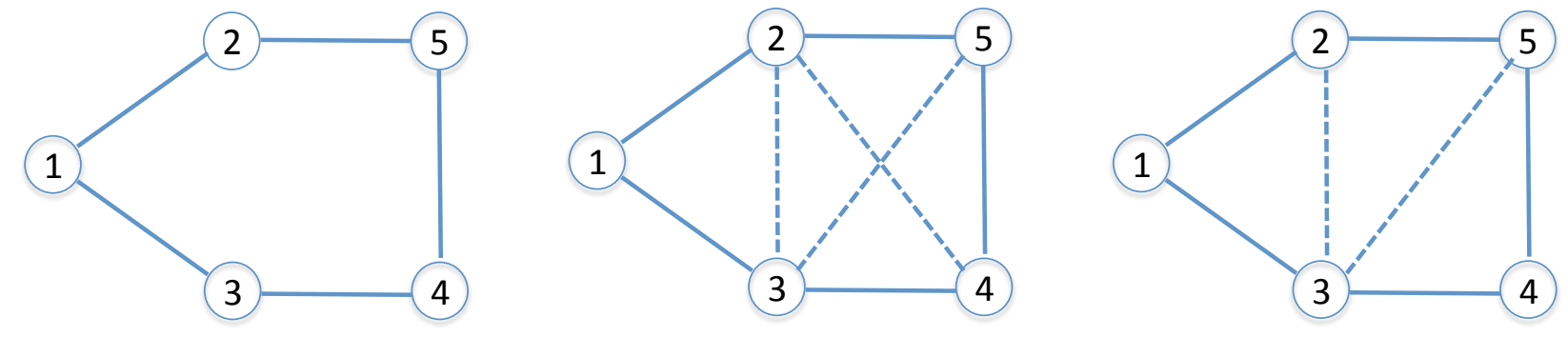

$$
X_{G}=\left[\begin{array}{lllll}
x_{11} & x_{12} & x_{13} & & \\
x_{21} & x_{22} & & & x_{25} \\
x_{31} & & x_{33} & x_{34} & \\
& & x_{43} & x_{44} & x_{45} \\
& x_{52} & & x_{54} & x_{55}
\end{array}\right]
$$

$$
X_{F}=\left[\begin{array}{lllll}
x_{11} & x_{12} & x_{13} & & \\
x_{21} & x_{22} & x_{23} & x_{24} & x_{25} \\
x_{31} & x_{32} & x_{33} & x_{34} & x_{35} \\
\hdashline & x_{42} & x_{43} & x_{44} & x_{45} \\
& x_{52} & x_{53} & x_{54} & x_{55}
\end{array}\right]
$$

$$
X_{F}=\left[\begin{array}{llll|l}
x_{11} & x_{12} & x_{13} & & \\
x_{21} & x_{22} & x_{23} & & \\
x_{31} & x_{32} & x_{33} & x_{34} & x_{35} \\
\hline & & x_{43} & x_{44} & x_{45} \\
& x_{52} & x_{53} & x_{54} & x_{55}
\end{array}\right]
$$

\section{(a)}

(b)

(c)

Fig. 3: Chordal extensions of $G$. (a) Graph $G$ and the partial matrix $X_{G}$. (b) A chordal extension $F$ and its $X_{F}$ that have 2 maximal cliques, $q_{1}:=(1,2,3)$ and $q_{2}:=(2,3,4,5)$. These cliques share two nodes, 2 and 3. The corresponding $X_{F}\left(q_{1}\right)$ and $X_{F}\left(q_{2}\right)$ are outlined in $X_{F}$ with the overlapping entries shaded in green. The chordal relaxation based on this $F$ requires 4 decoupling variables $u_{j k}$. (c) Another chordal extension $F$ and its $X_{F}$ that have 3 maximal cliques, outlined and shaded in blue in $X_{F}$. The chordal relaxation based on this $F$ requires 8 decoupling variables $u_{j k}$.

Define the $7 \times 7$ block-diagonal matrix

$$
X^{\prime}:=\left[\begin{array}{cc}
X_{F}^{\prime}\left(q_{1}\right) & 0 \\
0 & X_{F}\left(q_{2}\right)
\end{array}\right]
$$

Then the chordal relaxation (43) can be written in the standard form (41) in terms of these $7 \times 7$ blockdiagonal Hermitian matrices:

$$
\begin{aligned}
\min _{X^{\prime} \in \mathbb{S}^{7}} & \operatorname{tr} C_{0}^{\prime} X^{\prime} \\
\text { subject to } & \operatorname{tr} C_{l}^{\prime} X^{\prime} \leq b_{l}, \quad l=1, \ldots, L \\
& \operatorname{tr} C_{r}^{\prime} X^{\prime}=0, \quad r=1,2,3,4 \\
& X^{\prime} \succeq 0
\end{aligned}
$$

for appropriate choices of $C_{l}^{\prime}, l=0, \ldots, L$. The constraint $X^{\prime} \succeq 0$ in (46d) is equivalent to the requirement 
(45) on its submatrices and $C_{r}^{\prime}$ in (46c) is chosen to enforce the requirement (44). Hence the chordal relaxation (43) is indeed an SDP.

Remark 11: There are two conflicting factors in choosing a good chordal extension $F$. First an $F$ that contains fewer number of maximal cliques $q$ generally involves larger cliques, leading to larger submatrices $X_{F}(q)$; for example the complete graph $F$ has a single maximal clique but the corresponding $X_{F}(q)=X$ has $n^{2}$ entries and the chordal relaxation (43) offers no computational advantage over solving (in fact it is exactly) the original SDP (41). This argues for a chordal extension $F$ with smaller, possibly more, maximal cliques $q$. Second, however, having more maximal cliques $q$ tends to require more decoupling variables $u_{j k}$. Every decoupling variable $u_{j k}$ introduces an extra equality constraint in (46c), thus increasing the required computational effort. For instance the transformed problem based on the chordal extension in Figure 3(b) involves 2 maximal cliques of sizes 3 and 4, and 4 additional equality constraints in (46c). The transformed problem based on the chordal extension in Figure 3(c), on the other hand, requires 3 maximal cliques each of size 3 , and 8 additional equality constraints.

In summary even though the ambient dimension of the new variable $X^{\prime}$ is generally larger than that of the original $n \times n$ matrix variable $X(7 \times 7$ as opposed to $5 \times 5$ for the example in Figure 3(b)), the chordal relaxation (43) can typically be solved much more efficiently than SDP (41) if $G$ is large and sparse; for OPF examples, see [30], [31]. Choosing a good chordal extension $F$ of $G$ is important but nontrivial. See [63], [64] for methods to compute efficient chordal extensions and sparse SDP solutions.

\section{APPENDIX}

\section{IX: PROOFS OF MAIN RESULTS}

\section{A. Proof of Theorem 1: equivalence}

Proof: Fix any injections $s \in \mathbb{C}^{n+1}$. It suffices to show that there is a bijection between the set of $V$ that satisfies (1) and the set of $\tilde{x}:=(S, I, \tilde{V})$ that satisfies (2). Here we use $V$ to denote a power flow solution in $\mathbb{V}(s)$ and $\tilde{V}$ to denote a component of a power flow solution $\tilde{x}$ in $\tilde{\mathbb{X}}(s)$. We now exhibit a function $g$ that maps $V$ to $\tilde{x}$ and its inverse $g^{-1}$.

To construct $g$ fix any $V$ that satisfies (1). Define $\tilde{V}(V):=V$ and $I(V)$ by (2b). Then define $S_{j k}(V)$ by (2c) (only) for $j \rightarrow k \in \tilde{E}$. This specifies $\tilde{x}:=g(V):=(S(V), I(V), \tilde{V}(V))$. We only need to show that $\tilde{x}$ satisfies (2a). For each $i \rightarrow j \in \tilde{E}$ we have by construction

$$
S_{i j}(V)-z_{i j}\left|I_{i j}(V)\right|^{2}=y_{i j}^{H} V_{i}\left(V_{i}-V_{j}\right)^{H}-y_{i j}^{H}\left|V_{i}-V_{j}\right|^{2}=-y_{i j}^{H} V_{j}\left(V_{j}-V_{i}\right)^{H}
$$

Hence for each $j \in N^{+}$

$$
\begin{aligned}
\sum_{k: j \rightarrow k} S_{j k}(V)-\sum_{i: i \rightarrow j}\left(S_{i j}(V)-z_{i j}\left|I_{i j}(V)\right|^{2}\right) & =\sum_{k: j \rightarrow k} y_{j k}^{H} V_{j}\left(V_{j}-V_{k}\right)^{H}+\sum_{i: i \rightarrow j} y_{i j}^{H} V_{j}\left(V_{j}-V_{i}\right)^{H} \\
& =\sum_{k: j \sim k} y_{j k}^{H} V_{j}\left(V_{j}^{H}-V_{k}^{H}\right)=s_{j}(V)
\end{aligned}
$$

which is $(2 a)$.

Conversely, given an $\tilde{x}:=(S, I, \tilde{V})$ that satisfies (2), define $V:=g^{-1}(\tilde{x}):=\tilde{V}$. That $\tilde{x}$ satisfies (2) implies that $g^{-1}$ as defined is indeed the inverse of $g$ above. Moreover (2a) implies that $V$ satisfies (1). 


\section{B. Proof of Theorem 2: rank-1 characterization}

The proof is from [31].

Proof: We will prove (1) $\Rightarrow(2) \Rightarrow(3) \Rightarrow(1)$. If $W$ is psd rank-1 then all its principle submatrices are psd and of rank 1 (the submatrix cannot be of rank 0 because, by assumption, $W_{j j}>0$ for all $j \in N^{+}$). This implies that its submatrix $W_{c(G)}$ is psd and rank-1. Hence $(1) \Rightarrow(2)$.

Fix a partial matrix $W_{c(G)}$ that is psd and rank-1 and consider its submatrix $W_{G}$. Since each link $(j, k) \in E$ is a clique of $c(G)$ the $2 \times 2$ principle submatrix $W_{G}(j, k)$ is psd and rank-1. Therefore to prove that $(2) \Rightarrow$ (3), it suffices to show that $W_{G}$ satisfies the cycle condition (13). We now prove the following statement by induction on $3 \leq k \leq n+1$ : for all cycles $\left(j_{1}, \ldots, j_{k}\right)$ of length $k$ in $c(G)$,

$$
\sum_{i=1}^{k} \angle\left[W_{G}\right]_{j_{i} j_{i+1}}=0 \bmod 2 \pi
$$

where $j_{k+1}:=j_{1}$. For $k=3$, a cycle $\left(n_{1}, n_{2}, n_{3}\right)$ is a clique of $c(G)$ and therefore the following principle submatrix of $W_{c(G)}$ :

$$
W_{c(G)}\left(n_{1}, n_{2}, n_{3}\right):=\left[\begin{array}{lll}
{\left[W_{c(G)}\right]_{n_{1} n_{1}}} & {\left[W_{c(G)}\right]_{n_{1} n_{2}}} & {\left[W_{c(G)}\right]_{n_{1} n_{3}}} \\
{\left[W_{c(G)}\right]_{n_{2} n_{1}}} & {\left[W_{c(G)}\right]_{n_{2} n_{2}}} & {\left[W_{c(G)}\right]_{n_{2} n_{3}}} \\
{\left[W_{c(G)}\right]_{n_{3} n_{1}}} & {\left[W_{c(G)}\right]_{n_{3} n_{2}}} & {\left[W_{c(G)}\right]_{n_{3} n_{3}}}
\end{array}\right]
$$

defined on the cycle is psd rank- 1 . Hence $W_{c(G)}\left(n_{1}, n_{2}, n_{3}\right)=u u^{H}$ for some $u:=\left(u_{1}, u_{2}, u_{3}\right) \in \mathbb{C}^{3}$. Then

$$
\sum_{i=1}^{3} \angle\left[W_{G}\right]_{j_{i} j_{i+1}}=\angle\left[\left(u_{1} u_{2}^{H}\right)\left(u_{2} u_{3}^{H}\right)\left(u_{3} u_{1}^{H}\right)\right]=0 \bmod 2 \pi
$$

Suppose (47) holds for all cycles in $c(G)$ of length up to $k>3$. Consider now a cycle $\left(j_{1}, \ldots, j_{k+1}\right)$ of length $k+1$ in $c(G)$. Since $c(G)$ is chordal there is a chord, say, $\left(j_{1}, j_{l}\right) \in E$ for some $1<l<k+1$. Since both cycles $\left(j_{1}, \ldots, j_{l}\right)$ and $\left(j_{1}, j_{l}, \ldots, j_{k+1}\right)$ satisfy $(47)$ we have

$$
\begin{aligned}
& \sum_{i=1}^{l-1} \angle\left[W_{G}\right]_{j_{i} j_{i+1}}+\angle\left[W_{G}\right]_{j_{l} j_{1}}=0 \bmod 2 \pi \\
& \angle\left[W_{G}\right]_{j_{1} j_{l}}+\sum_{i=l}^{k+1} \angle\left[W_{G}\right]_{j_{i} j_{i+1}}=0 \bmod 2 \pi
\end{aligned}
$$

where $j_{k+2}:=j_{1}$. Since $W_{G}$ is Hermitian, adding the above equations yields

$$
\sum_{i=1}^{k+1} \angle\left[W_{G}\right]_{j_{i} j_{i+1}}=0 \bmod 2 \pi
$$

proving (47) for $k+1$. This completes the proof of $(2) \Rightarrow(3)$.

For (3) $\Rightarrow(1)$, fix any partial matrix $W_{G}$ that is $2 \times 2$ psd rank-1 and satisfies the cycle condition (13). We now construct a psd rank-1 completion $W$ of $W_{G}$, by constructing a vector $V \in \mathbb{C}^{n+1}$ such that $W=V V^{H}$. Let

$$
\left|V_{j}\right|:=\sqrt{\left[W_{G}\right]_{j}}
$$


Without loss of generality let $\angle V_{0}=0^{\circ}$; for $j=1, \ldots, n$, set

$$
\angle V_{j}:=-\sum_{(i, k) \in \mathbb{P}_{j}} \angle\left[W_{G}\right]_{i k}
$$

where $\mathbb{P}_{j}$ is any path from node 0 to node $j$. This is well defined because $W_{G}$ satisfies the cycle condition (13). It can be checked that $W=V V^{H}$ is indeed a psd rank-1 completion of $W_{G}$. This completes the proof.

\section{Proof of Corollary 3: uniqueness of completion}

The proof is from [57].

Proof: The proof of Theorem 2 shows that given a partial matrix $W_{c(G)} \in \mathbb{W}_{c(G)}$, the (unique) submatrix $W_{G}$ of $W_{c(G)}$ has a psd rank-1 completion $W \in \mathbb{W}$. Therefore to prove the corollary it suffices to prove that any partial matrix $W_{G} \in \mathbb{W}_{G}$ has a unique psd rank-1 completion $W \in \mathbb{W}$. To this end fix a $W_{G} \in \mathbb{W}_{G}$ and suppose there are two psd rank-1 completions $U U^{H}$ and $V V^{H}$ in $\mathbb{W}$. Clearly $\left|U_{j}\right|=\left|V_{j}\right|=\sqrt{\left[W_{G}\right]_{j j}}$ for all $j \in N^{+}$; moreover $\left(\angle U_{j}, j \in N^{+}\right)$and $\left(\angle V_{j}, j \in N^{+}\right)$are solutions of

$$
B \theta=\beta \quad \bmod 2 \pi
$$

where $B$ is the $m \times n$ reduced incidence matrix defined in Section V-A and $\beta \in \mathbb{R}^{m}$ with $\beta_{j k}:=\angle\left[W_{G}\right]_{j k}$. Since $G$ is connected, $m \geq n$ and hence the solution $\theta$ is unique. Therefore $U=V$ and the psd rank-1 completion of $W_{G}$ is unique.

\section{Proof of Theorem 5: BIM feasible sets}

Proof: First $\mathbb{V} \sqsubseteq \mathbb{W}^{+} \sqsubseteq \mathbb{W}_{c(G)}^{+} \sqsubseteq \mathbb{W}_{G}^{+}$follows from Theorem 4 and the definitions of $\mathbb{W}^{+}, \mathbb{W}_{c(G)}^{+}, \mathbb{W}_{G}^{+}$ (recall that by assumption the cost function $C$ depends on $V, W, W_{c(G)}$ only through the submatrix $W_{G}$ ). Since $c(G)$ is chordal, [62, Theorem 7] implies that every $W_{c(G)}$ in $\mathbb{W}_{c(G)}^{+}$has a psd completion $W$ in $\mathbb{W}^{+}$, i.e., $\mathbb{W}_{c(G)}^{+} \sqsubseteq \mathbb{W}^{+}$. Hence $\mathbb{W}^{+} \simeq \mathbb{W}_{c(G)}^{+}$.

Suppose $G$ is a tree and consider any chordal extension $c(G)$. We need to show that $\mathbb{W}_{G}^{+} \sqsubseteq \mathbb{W}_{c(G)}^{+}$, i.e., given any $W_{G} \in \mathbb{W}_{G}^{+}$there is a $W_{c(G)} \in \mathbb{W}_{c(G)}^{+}$with the same cost. Since $G$ is itself chordal, [62, Theorem 7] implies that $W_{G}$ has a psd completion $W$ in $\mathbb{W}^{+}$. The submatrix $W_{c(G)}$ of $W$ defined on $c(G)$ is the desired partial matrix in $\mathbb{W}_{c(G)}^{+}$with the same cost. This proves $\mathbb{W}_{G}^{+} \sqsubseteq \mathbb{W}_{c(G)}^{+}$and hence $\mathbb{W}_{G}^{+} \simeq \mathbb{W}_{c(G)}^{+}$for radial networks.

\section{E. Proof of Theorem 7: BFM feasible sets}

That $\mathbb{X} \subseteq \mathbb{X}_{n c}$ follows from their definitions. Hence we only prove $\tilde{\mathbb{X}} \equiv \mathbb{X}$, following [24].

Fix any $x:=(S, \ell, v, s)$ in $\mathbb{X}_{n c}$ and the corresponding $\beta(x)$ defined in (25). Consider the cycle condition which is an equation in the variable $(\theta, k)$ :

$$
B \theta=\beta(x)+2 \pi k
$$

where $k \in \mathbb{N}^{m}$ is an integer vector. Since $G$ is connected, $m \geq n$ and $\operatorname{rank}(B)=n$. Hence, given any $k$, there is at most one $\theta$ that solves (48). Conversely, given a solution $\theta$, the corresponding $k$ is unique, i.e., if the 
pairs $(\theta, k)$ and $\left(\theta, k^{\prime}\right)$ are both solutions of (48), then $k=k^{\prime}$. We therefore sometimes refer to a solution $(\theta, k)$ simply as $\theta$ and write $k$ as $k(\theta)$ when we want to emphasize its dependence on $\theta$. Given any pair $(\theta, k)$ with $\theta \in(-\pi, \pi]^{n}$, define its equivalence class as

$$
\sigma(\theta, k):=\left\{(\theta+2 \pi \alpha, k+B \alpha) \mid \alpha \in \mathbb{N}^{n}\right\}
$$

Using the connectedness of $G$ and the definition of $B$, one can argue that $\alpha$ must be an integer vector for $k+B \alpha$ to be integral. We say $\sigma(\theta, k)$ is a solution of (48) if every vector in $\sigma(\theta, k)$ is a solution of (48), and $\sigma(\theta, k)$ is the unique solution of (48) if it is the only equivalence class of solutions. The lemma below implies that if a solution of (48) exists then it is unique.

We abuse (to simplify) notation and use $\theta$ to denote either an $n$-dimensional vector $\theta:=\left(\theta_{j}, j \in N\right)$ or an $(n+1)$-dimensional vector $\theta:=\left(\theta_{j}, j \in N^{+}\right)$with $\theta_{0}:=\angle V_{0}:=0^{\circ}$, depending on the context. For each $\theta \in(-\pi, \pi]^{n+1}$, define the mapping $\tilde{h}_{\theta}(S, \ell, v, s)=(S, I, V, s)$ from $\mathbb{R}^{3(m+n+1)}$ to $\mathbb{C}^{2(m+n+1)}$ by:

$$
\begin{aligned}
V_{j} & :=\sqrt{v_{j}} e^{\mathbf{i} \theta_{j}}, \quad j \in N^{+} \\
I_{j k} & :=\sqrt{\ell_{j k}} e^{\mathbf{i}\left(\theta_{j}-\angle S_{j k}\right)}, j \rightarrow k \in \tilde{E}
\end{aligned}
$$

The proof of Theorem 7 relies on the following lemma that gives a necessary and sufficient condition on $\theta$ for $\tilde{x}:=\tilde{h}_{\theta}(x)$ to be in $\tilde{\mathbb{X}}$.

Lemma 14: Fix any $x:=(S, \ell, v, s)$ in $\mathbb{X}_{n c}$ and the corresponding $\beta(x)$ defined in (25). Then

1) $\tilde{x}:=\tilde{h}_{\theta}(x)$ is in $\tilde{\mathbb{X}}$ if and only if $(\theta, k(\theta))$ solves (48).

2) there is at most one $\sigma(\theta, k)$ with $\theta \in(-\pi, \pi]^{n}$, that is the unique solution of (48) when it exists.

Proof of Lemma 14: To prove the first claim, suppose $(\theta, k)$ is a solution of (26) for some $k=k(\theta)$. We need to show that (24), (48) together with (49) imply (2). Now (24a) is equivalent to (2a). Moreover (24c) and (49) imply (2c). To prove (2b), substitute (2c) into (48) to get

$$
\theta_{i}-\theta_{j}=\angle\left(v_{i}-z_{i j}^{H} V_{i} I_{i j}^{H}\right)+2 \pi k_{i j}=\angle V_{i}\left(V_{i}-z_{i j} I_{i j}\right)^{H}+2 \pi k_{i j}
$$

Hence

$$
\angle V_{j}=\theta_{j}=\angle\left(V_{i}-z_{i j} I_{i j}\right)-2 \pi k_{i j}
$$

From (24b), we have

$$
\begin{aligned}
\left|V_{j}\right|^{2} & =\left|V_{i}\right|^{2}+\left|z_{i j}\right|^{2}\left|I_{i j}\right|^{2}-\left(z_{i j} S_{i j}^{H}+z_{i j}^{H} S_{i j}\right) \\
& =\left|V_{i}\right|^{2}+\left|z_{i j}\right|^{2}\left|I_{i j}\right|^{2}-\left(z_{i j} V_{i}^{H} I_{i j}+z_{i j}^{H} V_{i} I_{i j}^{H}\right) \\
& =\left|V_{i}-z_{i j} I_{i j}\right|^{2}
\end{aligned}
$$

where the second equality follows from (2c). This and (50) imply $V_{j}=V_{i}-z_{i j} I_{i j}$ which is (2b). This completes the proof that if $(\theta, k(\theta))$ is a solution of (48) then $\tilde{x}:=\tilde{h}_{\theta}(x)$ lies in $\tilde{\mathbb{X}}$.

Conversely suppose $h_{\theta}(x) \in \mathbb{X}$. From (2b)-(2c), we have $V_{i} V_{j}^{H}=\left|V_{i}\right|^{2}-z_{i j}^{H} S_{i j}$. Then $\theta_{i}-\theta_{j}=\beta_{i j}+2 \pi k_{i j}$ for some integer $k_{i j}=k_{i j}(\theta)$. Hence $(\theta, k)$ solves (48).

For the second claim, the discussion preceding the lemma shows that, given any $k \in \mathbb{N}^{m}$, there is at most 
one $\theta$ that satisfies (48). If no such $\theta$ exists for any $k \in \mathbb{N}^{m}$, then (48) has no solution $(\theta, k)$. If (48) has a solution $(\theta, k)$, then clearly $(\theta+2 \pi \alpha, k+B \alpha)$ are also solutions for all integer vectors $\alpha \in \mathbb{N}^{n}$. Hence we can assume without loss of generality that $\theta \in(-\pi, \pi]^{n}$. We claim that $\sigma(\theta, k)$ is the unique solution of (48). Otherwise, there is an $(\tilde{\theta}, \tilde{k}) \notin \sigma(\theta, k)$ with $B \tilde{\theta}=\beta+2 \pi \tilde{k}$. Then $B(\tilde{\theta}-\theta)=2 \pi(\tilde{k}-k)$, or

$$
\tilde{k}=k+B \alpha \quad \text { where } \quad \alpha:=\frac{1}{2 \pi}(\tilde{\theta}-\theta)
$$

Note that both $k$ and $\tilde{k}$ are integer vectors in $\mathbb{N}^{m}$. Using the connectedness of $\mathrm{G}$ and the definition of $B$, one can argue that $\alpha$ must be an integer vector in $\mathbb{N}^{n}$ for $k+B \alpha$ to be integral. Then $\tilde{\theta}=\theta+2 \pi \alpha$ for some integer vector $\alpha$. This means $(\tilde{\theta}, \tilde{k}) \in \sigma(\theta, k)$, a contradiction.

Proof of Theorem 7: We now show that $\tilde{\mathbb{X}} \equiv \mathbb{X}$ by explicitly specifying a bijection between these two sets. Recall the mapping $h: \tilde{\mathbb{X}} \rightarrow \mathbb{X}$ defined by $h(S, I, V, s)=(S, \ell, v, s)=: x$ with $\ell_{j}:=\left|I_{j}\right|^{2}$ and $v_{j}:=\left|V_{j}\right|^{2}$. Clearly it maps every $\tilde{x} \in \tilde{\mathbb{X}}$ to an $x$ that is in $\mathbb{X}$.

To construct its inverse, consider the family of mappings $\tilde{h}_{\theta}$ defined in (49) from $\mathbb{R}^{3(m+n+1)}$ to $\mathbb{C}^{2(m+n+1)}$, parameterized by every $\theta \in \mathbb{R}^{n}$. Lemma $14(1)$ implies that, given any $x$ in $\mathbb{X}$, if we use a specific $\theta$, namely $\theta(x)$ that is a solution of (48), then $\tilde{h}_{\theta(x)}(x)$ maps $x$ to an $\tilde{x}$ in $\tilde{\mathbb{X}}$. Moreover if we restrict $\theta(x)$ to $(-\pi, \pi]^{n+1}$ then $\tilde{h}_{\theta(x)}(x)$ is uniquely defined. Consider then the overall mapping $\tilde{h}_{\theta(x)}(x)$ from $x \in \mathbb{X}$ to an $\tilde{x} \in \tilde{\mathbb{X}}$ with $\theta(x) \in(-\pi, \pi]^{n+1}$. Note that for each $x \in \mathbb{X}$, this mapping selects a possibly different mapping from the family of mappings $\tilde{h}_{\theta}, \theta \in \mathbb{R}^{n+1}$ with $\theta_{0}(x):=\angle V_{0}:=0^{\circ}$. Clearly $h\left(\tilde{h}_{\theta(x)}(x)\right)=x$ and $\tilde{h}_{\theta(h(\tilde{x}))}(h(\tilde{x}))=\tilde{x}$. This means that $h^{-1}(\cdot):=\tilde{h}_{\theta(\cdot)}(\cdot)$ is an inverse of $h(\cdot)$.

This completes the proof of Theorem 7.

Notice that the inverse $h^{-1}(\cdot):=h_{\theta(\cdot)}(\cdot)$ is defined by the function $\theta(\cdot)$. Even though $\theta$ is not unique (if $\theta$ defines an inverse of $h$, then $\theta+2 \pi \alpha$ also defines a valid inverse), the inverse $h^{-1}$ is unique because both $\theta$ and $\theta+2 \pi \alpha$ map a point $x$ in $\mathbb{X}$ to the same point $\tilde{x} \in \tilde{\mathbb{X}}$ (see (49)). Hence $\tilde{\mathbb{X}} \equiv \mathbb{X}$ is indeed well defined. When we fix $\theta$ to be in $(-\pi, \pi]^{n}$, it fixes a unique $\theta$ that defines $h^{-1}$ as well.

\section{F. Proof of Theorem 8: BFM cycle condition}

The proof is from [24].

Proof: Since $m \geq n$ and $\operatorname{rank}(B)=n$, we can always find $n$ linearly independent rows of $B$ to form a basis. The choice of this basis corresponds to choosing a spanning tree of $G$, which always exists since $G$ is connected [77, Chapter 5]. Assume without loss of generality that the first $n$ rows is such a basis and partition $B$ and $\beta$ accordingly. Then Lemma 14 implies that $h_{\theta_{*}}(x) \in \mathbb{X}$ (in particular, $x$ satisfies the cycle condition (26)) with $\theta_{*} \in(-\pi, \pi]^{n}$ if and only if $\left(\theta_{*}, k\left(\theta_{*}\right)\right)$ is the unique solution of

$$
\left[\begin{array}{l}
B_{T} \\
B_{\perp}
\end{array}\right] \theta=\left[\begin{array}{l}
\beta_{T} \\
\beta_{\perp}
\end{array}\right]+2 \pi\left[\begin{array}{l}
k_{T} \\
k_{\perp}
\end{array}\right]
$$

with $\theta \in(-\pi, \pi]^{n}$. Since $T$ is a spanning tree, the $n \times n$ submatrix $B_{T}$ is invertible. Moreover (51) has a unique solution if and only if $B_{\perp} B_{T}^{-1}\left(\beta_{T}+2 \pi k_{T}\right)=\beta_{\perp}+2 \pi k_{\perp}$, or if and only if

$$
B_{\perp} B_{T}^{-1} \beta_{T}=\beta_{\perp}+2 \pi \hat{k}_{\perp}
$$


for some integer vector $\hat{k}_{\perp}:=k_{\perp}-B_{\perp} B_{T}^{-1} k_{T}$. ((53) below implies that $\hat{k}_{\perp}$ is indeed an integer vector.)

We can assume without loss of generality that the orientation of the network graph $\tilde{G}$ is such that all the links in $T$ are directed away from the root node 0 . Let $\mathbb{P}(i \rightsquigarrow j)$ denote the unique path from node $i$ to node $j$ in $T$; in particular, $\mathbb{P}(0 \rightsquigarrow j)$ consists of links all with the same orientation as the path and $\mathbb{P}(j \rightsquigarrow 0)$ of links all with the opposite orientation. Then it can be verified directly that

$$
\left[B_{T}^{-1}\right]_{i e}:= \begin{cases}-1 & \text { if link } e \text { is in } \mathbb{P}(0 \rightsquigarrow i) \\ 0 & \text { otherwise }\end{cases}
$$

Hence $B_{T}^{-1} \beta_{T}$ represents the (negative of the) sum of angle differences on the path $\mathbb{P}(0 \rightsquigarrow i)$ for each node $i \in T$ :

$$
\left[B_{T}^{-1} \beta_{T}\right]_{i}=\sum_{e}\left[B_{T}^{-1}\right]_{i e}\left[\beta_{T}\right]_{e}=-\sum_{e \in \mathbb{P}(0 \rightsquigarrow i)}\left[\beta_{T}\right]_{e}
$$

Hence $B_{\perp} B_{T}^{-1} \beta_{T}$ is the sum of angle differences from node $i$ to node $j$ along the unique path in $T$, for every link $i \rightarrow j \in \tilde{E} \backslash \tilde{E}_{T}$ not in the tree $T$. To see this, we have, for each link $e:=i \rightarrow j \in \tilde{E} \backslash \tilde{E}_{T}$,

$$
\left[B_{\perp} B_{T}^{-1} \beta_{T}\right]_{e}=\left[B_{T}^{-1} \beta_{T}\right]_{i}-\left[B_{T}^{-1} \beta_{T}\right]_{j}=\sum_{e^{\prime} \in \mathbb{P}(0 \rightsquigarrow j)}\left[\beta_{T}\right]_{e^{\prime}}-\sum_{e^{\prime} \in \mathbb{P}(0 \rightsquigarrow i)}\left[\beta_{T}\right]_{e^{\prime}}
$$

Therefore (52) implies, for each link $i \rightarrow j \in \tilde{E} \backslash \tilde{E}_{T}$,

$$
\beta_{i j}-\left(\sum_{e^{\prime} \in \mathbb{P}(0 \rightsquigarrow j)}\left[\beta_{T}\right]_{e^{\prime}}-\sum_{e^{\prime} \in \mathbb{P}(0 \rightsquigarrow i)}\left[\beta_{T}\right]_{e^{\prime}}\right)=-2 \pi \hat{k}_{\perp}
$$

Consider the basis cycle $c(i, j)$ defined by such a link $i \rightarrow j$ outside the spanning tree $T$ [77, Chapter 5]. Using the definition of $\tilde{\beta}$ we have

$$
\begin{aligned}
\sum_{e^{\prime} \in c(i, j)} \tilde{\beta}_{e^{\prime}} & =\tilde{\beta}_{i j}+\sum_{e^{\prime} \in \mathbb{P}(j \rightsquigarrow 0)} \tilde{\beta}_{e^{\prime}}+\sum_{e^{\prime} \in \mathbb{P}(0 \rightsquigarrow i)} \tilde{\beta}_{e^{\prime}} \\
& =\beta_{i j}-\sum_{e^{\prime} \in \mathbb{P}(0 \rightsquigarrow j)} \beta_{e^{\prime}}+\sum_{e^{\prime} \in \mathbb{P}(0 \rightsquigarrow i)} \beta_{e^{\prime}}=-2 \pi\left[\hat{k}_{\perp}\right]_{i j}
\end{aligned}
$$

i.e., $\sum_{e^{\prime} \in c(i, j)} \tilde{\beta}_{e^{\prime}}=0 \bmod 2 \pi$. Since this holds for all basis cycles, it holds for all undirected cycles. Therefore the cycle conditions (26) and (28) are equivalent.

Finally consider the unique solution $\left(\theta_{*}, k\left(\theta_{*}\right)\right)$ of (48) with $\theta_{*} \in(-\pi, \pi]^{n}$. By (51) we have $\theta_{*}=$ $B_{T}^{-1} \beta_{T}+2 \pi B_{T}^{-1} k_{T}\left(\theta_{*}\right)$. The definition of $k\left(\theta_{*}\right)$ and the fact $\theta_{*} \in(-\pi, \pi]^{n}$ imply that $\theta_{*}=\mathscr{P}\left(B_{T}^{-1} \beta_{T}\right)$; see the discussion preceding Lemma 14. This completes the proof of Theorem 8.

\section{G. Proof of Theorem 9: radial networks}

The proof is from [24].

Proof: Theorem 7 and the definition of $\mathbb{X}^{+}$imply $\tilde{\mathbb{X}} \equiv \mathbb{X} \subseteq \mathbb{X}_{n c} \subseteq \mathbb{X}^{+}$. If the network $\tilde{G}=T$ is a tree then $B=B_{T}$ is $n \times n$ and invertible. Hence every $x \in \mathbb{X}_{n c}$ satisfies the cycle condition (26) and hence is in $\mathbb{X}$. Therefore $\mathbb{X}=\mathbb{X}_{n c}$. 


\section{H. Proof of Theorem 11: equivalence}

The proof is from [57], [31].

Proof: Consider the linear mapping $g: \mathbb{W}_{G}^{+} \rightarrow \mathbb{X}^{+}$defined right after Theorem 11 by $x=g\left(W_{G}\right)$ where

$$
\begin{aligned}
S_{j k} & :=y_{j k}^{H}\left(\left[W_{G}\right]_{j j}-\left[W_{G}\right]_{j k}\right), \quad j \rightarrow k \in \tilde{E} \\
\ell_{j k} & :=\left|y_{j k}\right|^{2}\left(\left[W_{G}\right]_{j j}+\left[W_{G}\right]_{k k}-\left[W_{G}\right]_{j k}-\left[W_{G}\right]_{k j}\right), \quad j \rightarrow k \in \tilde{E} \\
v_{j} & :=\left[W_{G}\right]_{j j}, \quad j \in N^{+} \\
s_{j} & :=\sum_{k: j \sim k} y_{j k}^{H}\left(\left[W_{G}\right]_{j j}-\left[W_{G}\right]_{j k}\right), \quad j \in N^{+}
\end{aligned}
$$

and the mapping $g^{-1}: \mathbb{X}^{+} \rightarrow \mathbb{W}_{G}^{+}$with $W_{G}=g^{-1}(x)$ where

$$
\begin{aligned}
& {\left[W_{G}\right]_{j j}:=v_{j}, \quad j \in N^{+}} \\
& {\left[W_{G}\right]_{j k}:=v_{j}-z_{j k}^{H} S_{j k}=\left[W_{G}\right]_{k j}^{H}, \quad j \rightarrow k \in \tilde{E}}
\end{aligned}
$$

We will prove that $g$ and $g^{-1}$ are indeed inverses of each other in three steps: (1) $g$ maps every point $W_{G} \in \mathbb{W}_{G}^{+}$to a point in $\mathbb{X}^{+}$; (2) $g^{-1}$ maps every point $x \in \mathbb{X}^{+}$to a point in $\mathbb{W}_{G}^{+}$; and (3) $g\left(g^{-1}(x)\right)=x$ and $g^{-1}\left(g\left(W_{G}\right)\right)=W_{G}$. This defines a bijection between $\mathbb{W}_{G}^{+}$and $\mathbb{X}^{+}$and establishes $\mathbb{W}_{G}^{+} \equiv \mathbb{X}^{+}$. We will then prove the mappings $g\left(g^{-1}\right)$ restricted to $\mathbb{W}_{G}\left(\mathbb{W}_{n c}\right)$ and $\mathbb{X}\left(\mathbb{X}_{n c}\right)$ define the bijection between these sets.

Recall the sets:

$$
\begin{aligned}
\mathbb{W}_{G}^{+} & :=\left\{W_{G} \mid W_{G} \text { satisfies }(12), W_{G}(j, k) \succeq 0,(j, k) \in E\right\} \\
\mathbb{X}^{+} & :=\left\{x \in \mathbb{R}^{3(m+n+1)} \mid x \text { satisfies (3), (4), (24a),(24b),(29) }\right\}
\end{aligned}
$$

Step 1: $g\left(W_{G}\right) \in \mathbb{X}^{+}$. (12) and (55c)-(55d) imply (3) and (4). To prove (24a), we have for $j \in N^{+}$

$$
\begin{aligned}
& \sum_{i: i \rightarrow j}\left(S_{i j}-z_{i j} \ell_{i j}\right)+s_{j} \\
= & \sum_{i: i \rightarrow j}\left(y_{i j}^{H}\left(\left[W_{G}\right]_{i i}-\left[W_{G}\right]_{i j}\right)-y_{i j}^{H}\left(\left[W_{G}\right]_{i i}+\left[W_{G}\right]_{j j}-\left[W_{G}\right]_{i j}-\left[W_{G}\right]_{j i}\right)\right)+s_{j} \\
= & \sum_{i: i \rightarrow j}\left(-y_{i j}^{H}\left(\left[W_{G}\right]_{j j}-\left[W_{G}\right]_{j i}\right)\right)+\sum_{i: i \rightarrow j} y_{j i}^{H}\left(\left[W_{G}\right]_{j j}-\left[W_{G}\right]_{j i}\right)+\sum_{k: j \rightarrow k} y_{j k}^{H}\left(\left[W_{G}\right]_{j j}-\left[W_{G}\right]_{j k}\right) \\
= & \sum_{k: j \rightarrow k} S_{j k}
\end{aligned}
$$

as desired. To prove (24b), we have for $j \rightarrow \in \tilde{E}$

$$
\begin{aligned}
2 \operatorname{Re}\left(z_{j k}^{H} S_{j k}\right)-\left|z_{j k}\right|^{2} \ell_{j k} & =2 \operatorname{Re}\left(\left[W_{G}\right]_{j j}-\left[W_{G}\right]_{j k}\right)-\left(\left[W_{G}\right]_{j j}+\left[W_{G}\right]_{k k}-\left[W_{G}\right]_{j k}-\left[W_{G}\right]_{k j}\right) \\
& =\left(\left[W_{G}\right]_{j j}-\left[W_{G}\right]_{k k}\right)-\left[W_{G}\right]_{j k}^{H}+\left[W_{G}\right]_{k j} \\
& =v_{j}-v_{k}
\end{aligned}
$$


as desired. To prove (29) for each $j \rightarrow \in \tilde{E}$, use $\left[W_{G}\right]_{j j}\left[W_{G}\right]_{j k} \geq\left|\left[W_{G}\right]_{j k}\right|^{2}$ to get

$$
\begin{aligned}
v_{j} \ell_{j k} & =\left|y_{j k}\right|^{2}\left[W_{G}\right]_{j j}\left(\left[W_{G}\right]_{j j}+\left[W_{G}\right]_{k k}-\left[W_{G}\right]_{j k}-\left[W_{G}\right]_{k j}\right) \\
& \geq\left|y_{j k}\right|^{2}\left(\left[W_{G}\right]_{j j}^{2}+\left|\left[W_{G}\right]_{j k}\right|^{2}-\left[W_{G}\right]_{j j}\left[W_{G}\right]_{j k}-\left[W_{G}\right]_{j j}\left[W_{G}\right]_{j k}^{H}\right) \\
& =\left|S_{j k}\right|^{2}
\end{aligned}
$$

as desired. Hence $g$ maps every $W_{G} \in \mathbb{W}_{G}^{+}$to a point in $\mathbb{X}^{+}$.

Step 2: $g^{-1}(x) \in \mathbb{W}_{G}^{+}$. Clearly (56a) and (3) imply (12b). To prove (12a), we have for each $j \in N^{+}$

$$
\begin{aligned}
\sum_{k:(j, k) \in E} y_{j k}^{H}\left(\left[W_{G}\right]_{j j}-\left[W_{G}\right]_{j k}\right) & =\sum_{i: i \rightarrow j} y_{j i}^{H}\left(\left[W_{G}\right]_{j j}-\left[W_{G}\right]_{j i}\right)+\sum_{k: j \rightarrow k} y_{j k}^{H}\left(\left[W_{G}\right]_{j j}-\left[W_{G}\right]_{j k}\right) \\
& =\sum_{i: i \rightarrow j} y_{i j}^{H}\left(v_{j}-\left(v_{i}-z_{i j}^{H} S_{i j}\right)^{H}\right)+\sum_{k: j \rightarrow k} y_{j k}^{H}\left(v_{j}-\left(v_{j}-z_{j k}^{H} S_{j k}\right)\right) \\
& =\sum_{k: j \rightarrow k} S_{j k}-\sum_{i: i \rightarrow j} y_{i j}^{H}\left(v_{i}-v_{j}-z_{i j} S_{i j}^{H}\right) \\
& =\sum_{k: j \rightarrow k} S_{j k}-\sum_{i: i \rightarrow j} y_{i j}^{H}\left(2 \operatorname{Re}\left(z_{i j}^{H} S_{i j}\right)-\left|z_{i j}\right|^{2} \ell_{i j}-z_{i j} S_{i j}^{H}\right)
\end{aligned}
$$

where the second equality follows from (56) and the last equality from (24b). But

$$
\left(2 \operatorname{Re}\left(z_{i j}^{H} S_{i j}\right)-z_{i j} S_{i j}^{H}\right)=\left(z_{i j}^{H} S_{i j}+z_{i j} S_{i j}^{H}\right)-z_{i j} S_{i j}^{H}=z_{i j}^{H} S_{i j}
$$

and hence

$$
\sum_{k:(j, k) \in E} y_{j k}^{H}\left(\left[W_{G}\right]_{j j}-\left[W_{G}\right]_{j k}\right)=\sum_{k: j \rightarrow k} S_{j k}-\sum_{i: i \rightarrow j}\left(S_{i j}-z_{i j} \ell_{i j}\right)=s_{j}
$$

This and (4) imply (12a) as desired. To prove $W_{G}(j, k) \succeq 0$ for each $(j, k) \in E$, we have

$$
\begin{aligned}
{\left[W_{G}\right]_{j j}\left[W_{G}\right]_{k k}-\left|\left[W_{G}\right]_{j k}\right|^{2} } & =v_{j} v_{k}-\left|v_{j}-z_{j k}^{H} S_{j k}\right|^{2} \\
& =v_{j} v_{k}-\left(v_{j}^{2}+\left|z_{j k}\right|^{2}\left|S_{j k}\right|^{2}-2 v_{j} \operatorname{Re}\left(z_{j k}^{H} S_{j k}\right)\right) \\
& =v_{j}\left(v_{k}-v_{j}+2 \operatorname{Re}\left(z_{j k}^{H} S_{j k}\right)\right)-\left|z_{j k}\right|^{2}\left|S_{j k}\right|^{2} \\
& =\left|z_{j k}\right|^{2}\left(v_{j} \ell_{j k}-\left|S_{j k}\right|^{2}\right)
\end{aligned}
$$

where the last equality follows from (24b). Therefore $W_{G}(j, k) \succeq 0$ follows from (29) as desired. Hence $g^{-1}$ maps every point $x \in \mathbb{X}^{+}$to a point in $\mathbb{W}_{G}^{+}$.

Step 3: $g\left(g^{-1}(x)\right)=x$ and $g^{-1}\left(g\left(W_{G}\right)\right)=W_{G}$. The proof uses (55), (56), (24) and follows similar argument used in Steps 1 and 2, and is thus omitted. This completes the proof that $g$ and $g^{-1}$ are indeed inverses of each other and establishes $\mathbb{W}_{G}^{+} \equiv \mathbb{X}^{+}$.

From (57), we have

$$
v_{j} \ell_{j k}=\left|S_{j k}\right|^{2} \quad \text { if and only if } \quad\left[W_{G}\right]_{j j}\left[W_{G}\right]_{k k}=\left|\left[W_{G}\right]_{j k}\right|^{2}
$$

This implies that $g$ and $g^{-1}$ restricted to $\mathbb{W}_{n c}$ and $\mathbb{X}_{n c}$ respectively are inverses of each other as well, 
establishing $\mathbb{W}_{n c} \equiv \mathbb{X}_{n c}$.

Finally to prove $\mathbb{W} \equiv \mathbb{X}$ we need to show that the cycle conditions (13) and (26) are equivalent, i.e., $W_{G} \in \mathbb{W}_{n c}$ satisfies (13) if and only if $x:=g\left(W_{G}\right) \in \mathbb{X}_{n c}$ satisfies (26). Consider any cycle $c$. Using (56b) and the definition of $\tilde{\beta}(x)$ before Theorem 8 , we have

$$
\sum_{(j, k) \in c} \angle W_{j k}=\sum_{(j, k) \in c} \angle \tilde{\beta}_{j k}
$$

Theorem 8 therefore implies that the cycle conditions (13) and (26) are equivalent under $g$ and $g^{-1}$.

This completes the proof of Theorem 11.

\section{Proof of Lemma 12: voltage bound}

The proofs of 12(1)-(3) and Lemma 13 are obvious and omitted. The proof of Lemma 12(4) makes use of Lemma 13 and is provided here.

Proof of Lemma 12(3): Fix $v_{0}$ and an $s \in \mathbb{R}^{2(n+1)}$. Let $x:=(S, \ell, v)$ and $x^{\text {lin }}:=\left(S^{\text {lin }}, \ell^{\text {lin }}, v^{\text {lin }}\right)$ be solutions of (33) and (37) respectively with the given $v_{0}$ and $s$, when $\tilde{G}$ is oriented so that all links point away from node 0 . We will prove $v \leq v^{\text {lin }}$, indirectly using Lemma 13.

Specifically consider the model where the network is oriented so that all links point towards node 0 and consider the solutions $\hat{x}:=(\hat{S}, \hat{\ell}, \hat{v})$ and $\hat{x}^{\text {lin }}:=\left(\hat{S}^{\text {lin }}, \hat{\ell}^{\text {lin }}, \hat{v}^{\text {lin }}\right)$ of (36) and (38) respectively with the given $v_{0}$ and $s$. Then Lemma 13 implies $\hat{v} \leq \hat{v}^{\text {lin }}$. We will prove that $v^{\text {lin }}=\hat{v}^{\text {lin }}$ and $v=\hat{v}$ and hence $v \leq v^{\text {lin }}$ as desired.

It is easy to see from (37) and (38) that $S_{j k}^{\operatorname{lin}}=-\hat{S}_{k j}^{\mathrm{lin}}$ and $v^{\mathrm{lin}}=\hat{v}^{\mathrm{lin}}$.

To show $v=\hat{v}$, define the following functions:

$$
\begin{aligned}
S_{k j}(\hat{S}, \hat{\ell}, \hat{v}) & :=-\left(\hat{S}_{j k}-z_{j k} \hat{\ell}_{j k}\right), j \rightarrow k \in E \\
\ell_{k j}(\hat{S}, \hat{\ell}, \hat{v}) & :=\hat{\ell}_{j k}, \quad j \rightarrow k \in E \\
v_{j}(\hat{S}, \hat{\ell}, \hat{v}) & :=\hat{v}_{j}, \quad j \in N
\end{aligned}
$$

Note that $(S(\hat{x}), \ell(\hat{x}), v(\hat{x}))$ are functions of $\hat{x}$ that is a solution of (38). ${ }^{9}$ Substituting these functions into (36) yields

$$
\begin{aligned}
&-S_{i j}(\hat{x})+z_{j i} \hat{\ell}_{j i}=-\sum_{k: k \rightarrow j} S_{j k}(\hat{x})+s_{j}, \quad j \in N^{+} \\
& v_{k}(\hat{x})-v_{j}(\hat{x})= 2 \operatorname{Re}\left(z_{k j}^{H}\left(-S_{j k}(\hat{x})+z_{k j} \hat{\ell}_{k j}\right)\right) \\
&-\left|z_{k j}\right|^{2} \hat{\ell}_{k j}, \quad k \rightarrow j \in \tilde{E} \\
& \ell_{j k}(\hat{x}) v_{k}(\hat{x})=\left|-S_{j k}(\hat{x})+z_{k j} \hat{\ell}_{k j}\right|^{2}, \quad k \rightarrow j \in \tilde{E}
\end{aligned}
$$

where as before $i$ in the above is the node on the unique path between node 0 and node $j$. Substituting

\footnotetext{
${ }^{9}$ Since $\hat{S}_{j k}$ represents the sending-end complex power from bus $j$ to bus $k,-S_{k j}(\hat{S}, \hat{\ell}, \hat{v})$ represents the received power by bus $k$ from bus $j$, net of the line loss $z_{j k} \ell_{j k}$.
} 
$\hat{\ell}_{k j}=\ell_{j k}(\hat{x})$ and rearranging, these equations become

$$
\begin{gathered}
\sum_{k: k \rightarrow j} S_{j k}(\hat{x})=S_{i j}(\hat{x})-z_{i j} \ell_{i j}(\hat{x})+s_{j}, \quad j \in N^{+} \\
v_{j}(\hat{x})-v_{k}(\hat{x})=2 \operatorname{Re}\left(z_{j k}^{H} S_{j k}(\hat{x})\right)-\left|z_{j k}\right|^{2} \ell_{j k}(\hat{x}) \\
k \rightarrow j \in \tilde{E} \\
\ell_{j k}(\hat{x}) v_{j}(\hat{x})=\left|S_{j k}(\hat{x})\right|^{2}, \quad k \rightarrow j \in \tilde{E}
\end{gathered}
$$

To obtain (60c) from (59), note that the right-hand side of (59) is

$$
\begin{aligned}
& \left|-S_{j k}(\hat{x})+z_{j k} \ell_{j k}(\hat{x})\right|^{2} \\
= & \left|S_{j k}(\hat{x})\right|^{2}+\left(\left|z_{j k}\right|^{2} \ell_{j k}(\hat{x})-2 \operatorname{Re}\left(z_{j k}^{H} S_{j k}(\hat{x})\right)\right) \ell_{j k}(\hat{x}) \\
= & \left|S_{j k}(\hat{x})\right|^{2}+\left(v_{k}(\hat{x})-v_{j}(\hat{x})\right) \ell_{j k}(\hat{x})
\end{aligned}
$$

where the second equality follows from (60b). This together with (59) imply (60c).

Notice that (60) is identical to (33) if we reverse the direction of the graph $\tilde{G}$. This means that the functions $(S(\hat{x}), \ell(\hat{x}), v(\hat{x}))$ are solutions of (33). Moreover the functions (58) can be compactly written in vector form as:

$$
\left[\begin{array}{l}
S \\
\ell \\
v
\end{array}\right](\hat{S}, \hat{\ell}, \hat{v})=\left[\begin{array}{ccc}
-I & Z & 0 \\
0 & I & 0 \\
0 & 0 & I
\end{array}\right]\left[\begin{array}{l}
\hat{S} \\
\hat{\ell} \\
\hat{v}
\end{array}\right]
$$

where the $m \times m$ matrix $Z$ is $\operatorname{diag}\left(z_{j k}, j \rightarrow k \in \tilde{E}\right)$. Clearly this mapping is invertible. This implies that there is a one-one correspondence between the solutions $x$ of (33) and the solutions $\hat{x}$ of (36). Moreover they have the same voltage magnitudes $v=\hat{v}$.

Hence Lemma 13(4) implies that $v=\hat{v} \leq \hat{v}^{\text {lin }}=v^{\text {lin }}$ as desired. 


\section{REFERENCES}

[1] J. Carpentier. Contribution to the economic dispatch problem. Bulletin de la Societe Francoise des Electriciens, 3(8):431-447, 1962.

[2] H.W. Dommel and W.F. Tinney. Optimal power flow solutions. Power Apparatus and Systems, IEEE Transactions on, PAS-87(10):18661876, Oct. 1968.

[3] J. A. Momoh. Electric Power System Applications of Optimization. Power Engineering. Markel Dekker Inc.: New York, USA, 2001.

[4] M. Huneault and F. D. Galiana. A survey of the optimal power flow literature. IEEE Trans. on Power Systems, 6(2):762-770, 1991.

[5] J. A. Momoh, M. E. El-Hawary, and R. Adapa. A review of selected optimal power flow literature to 1993. Part I: Nonlinear and quadratic programming approaches. IEEE Trans. on Power Systems, 14(1):96-104, 1999.

[6] J. A. Momoh, M. E. El-Hawary, and R. Adapa. A review of selected optimal power flow literature to 1993. Part II: Newton, linear programming and interior point methods. IEEE Trans. on Power Systems, 14(1):105 - 111, 1999.

[7] K. S. Pandya and S. K. Joshi. A survey of optimal power flow methods. J. of Theoretical and Applied Information Technology, 4(5):450-458, 2008.

[8] Stephen Frank, Ingrida Steponavice, and Steffen Rebennack. Optimal power flow: a bibliographic survey, I: formulations and deterministic methods. Energy Systems, 3:221-258, September 2012.

[9] Stephen Frank, Ingrida Steponavice, and Steffen Rebennack. Optimal power flow: a bibliographic survey, II: nondeterministic and hybrid methods. Energy Systems, 3:259-289, September 2013.

[10] Mary B. Cain, Richard P. O’Neill, and Anya Castillo. History of optimal power flow and formulations (OPF Paper 1). Technical report, US FERC, December 2012.

[11] Richard P. O'Neill, Anya Castillo, and Mary B. Cain. The IV formulation and linear approximations of the AC optimal power flow problem (OPF Paper 2). Technical report, US FERC, December 2012.

[12] Richard P. O'Neill, Anya Castillo, and Mary B. Cain. The computational testing of AC optimal power flow using the current voltage formulations (OPF Paper 3). Technical report, US FERC, December 2012.

[13] Anya Castillo and Richard P. O’Neill. Survey of approaches to solving the ACOPF (OPF Paper 4). Technical report, US FERC, March 2013.

[14] Anya Castillo and Richard P. O'Neill. Computational performance of solution techniques applied to the ACOPF (OPF Paper 5). Technical report, US FERC, March 2013.

[15] B Stott and O. Alsaç. Fast decoupled load flow. IEEE Trans. on Power Apparatus and Systems, PAS-93(3):859-869, 1974.

[16] O. Alsaç, J Bright, M Prais, and B Stott. Further developments in LP-based optimal power flow. IEEE Trans. on Power Systems, 5(3):697-711, 1990.

[17] Thomas J. Overbye, Xu Cheng, and Yan Sun. A comparison of the AC and DC power flow models for LMP calculations. In Proceedings of the 37th Hawaii International Conference on System Sciences, 2004.

[18] K. Purchala, L. Meeus, D. Van Dommelen, and R. Belmans. Usefulness of DC power flow for active power flow analysis. In Proc. of IEEE PES General Meeting, pages 2457-2462. IEEE, 2005.

[19] B. Stott, J. Jardim, and O. Alsaç. DC Power Flow Revisited. IEEE Trans. on Power Systems, 24(3):1290-1300, Aug 2009.

[20] Carleton Coffrin and Pascal Van Hentenryck. A linear-programming approximation of AC power flows. CoRR, abs/1206.3614, 2012.

[21] R.A. Jabr. Radial Distribution Load Flow Using Conic Programming. IEEE Trans. on Power Systems, 21(3):1458-1459, Aug 2006.

[22] X. Bai, H. Wei, K. Fujisawa, and Y. Wang. Semidefinite programming for optimal power flow problems. Int'l J. of Electrical Power \& Energy Systems, 30(6-7):383-392, 2008.

[23] Masoud Farivar, Christopher R. Clarke, Steven H. Low, and K. Mani Chandy. Inverter VAR control for distribution systems with renewables. In Proceedings of IEEE SmartGridComm Conference, October 2011.

[24] Masoud Farivar and Steven H. Low. Branch flow model: relaxations and convexification (parts I, II). IEEE Trans. on Power Systems, 28(3):2554-2572, August 2013.

[25] M. E. Baran and F. F Wu. Optimal Capacitor Placement on radial distribution systems. IEEE Trans. Power Delivery, 4(1):725-734, 1989.

[26] M. E Baran and F. F Wu. Optimal Sizing of Capacitors Placed on A Radial Distribution System. IEEE Trans. Power Delivery, 4(1):735-743, 1989.

[27] J. Lavaei and S. H. Low. Zero duality gap in optimal power flow problem. IEEE Trans. on Power Systems, 27(1):92-107, February 2012.

[28] X. Bai and H. Wei. A semidefinite programming method with graph partitioning technique for optimal power flow problems. Int'l J. of Electrical Power \& Energy Systems, 33(7):1309-1314, 2011.

[29] R. A. Jabr. Exploiting sparsity in sdp relaxations of the opf problem. Power Systems, IEEE Transactions on, 27(2):1138-1139, 2012.

[30] D. Molzahn, J. Holzer, B. Lesieutre, and C. DeMarco. Implementation of a large-scale optimal power flow solver based on semidefinite programming. IEEE Transactions on Power Systems, 28(4):3987-3998, November 2013. 
[31] Subhonmesh Bose, Steven H. Low, Thanchanok Teeraratkul, and Babak Hassibi. Equivalent relaxations of optimal power flow. IEEE Trans. Automatic Control, 2014.

[32] Z. Luo, W. Ma, A.M.C. So, Y. Ye, and S. Zhang. Semidefinite relaxation of quadratic optimization problems. Signal Processing Magazine, IEEE, 27(3):20 -34, May 2010.

[33] S. H. Low. Convex relaxation of optimal power flow, II: exactness. IEEE Trans. on Control of Network Systems, 2014. to appear.

[34] P. P. Varaiya, F. F. Wu, and J. W. Bialek. Smart operation of smart grid: Risk-limiting dispatch. Proceedings of the IEEE, 99(1):40-57, January 2011.

[35] M. Vrakopoulou, M. Katsampani, K. Margellos, J. Lygeros, and G. Andersson. Probabilistic security-constrained ac optimal power flow. In PowerTech (POWERTECH), 2013 IEEE Grenoble, pages 1-6, June 2013.

[36] Russell Bent, Daniel Bienstock, and Michael Chertkov. Synchronization-aware and algorithm-efficient chance constrained optimal power flow. In IREP Symposium - Bulk Power System Dynamics and Control (IREP), Rethymnon, Greece, August 2013.

[37] B. H. Kim and R. Baldick. Coarse-grained distributed optimal power flow. IEEE Trans. Power Syst., 12(2):932-939, May 1997.

[38] R. Baldick, B. H. Kim, C. Chase, and Y. Luo. A fast distributed implementation of optimal power flow. IEEE Trans. Power Syst., 14(3):858-864, August 1999.

[39] G. Hug-Glanzmann and G. Andersson. Decentralized optimal power flow control for overlapping areas in power systems. IEEE Trans. Power Syst., 24(1):327?336, February 2009.

[40] F. J. Nogales, F. J. Prieto, and A. J. Conejo. A decomposition methodology applied to the multi-area optimal power flow problem. Ann. Oper. Res., 120:99-116, 2003.

[41] Albert Lam, Baosen Zhang, and David N Tse. Distributed algorithms for optimal power flow problem. In IEEE CDC, pages 430-437, 2012.

[42] M. Kraning, E. Chu, J. Lavaei, and S. Boyd. Dynamic network energy management via proximal message passing. Foundations and Trends in Optimization, 1(2):70-122, 2013.

[43] K. Turitsyn, P. Sŭlc, S. Backhaus, and M. Chertkov. Options for control of reactive power by distributed photovoltaic generators. Proc. of the IEEE, 99(6):1063 -1073, June 2011.

[44] Albert Y.S. Lam, Baosen Zhang, Alejandro Domínguez-García, and David Tse. Optimal distributed voltage regulation in power distribution networks. arXiv, April 2012.

[45] Dzung T. Phan. Lagrangian duality and branch-and-bound algorithms for optimal power flow. Operations Research, 60(2):275-285, March/April 2012.

[46] A. Gopalakrishnan, A.U. Raghunathan, D. Nikovski, and L.T. Biegler. Global optimization of optimal power flow using a branch amp; bound algorithm. In Proc. of the 50th Annual Allerton Conference on Communication, Control, and Computing (Allerton), pages 609-616, Oct 2012.

[47] C. Josz, J. Maeght, P. Panciatici, and J. Ch. Gilbert. Application of the moment?sos approach to global optimization of the opf problem. arXiv, November 2013.

[48] H-D. Chiang and M. E. Baran. On the existence and uniqueness of load flow solution for radial distribution power networks. IEEE Trans. Circuits and Systems, 37(3):410-416, March 1990.

[49] Hsiao-Dong Chiang. A decoupled load flow method for distribution power networks: algorithms, analysis and convergence study. International Journal Electrical Power Energy Systems, 13(3):130-138, June 1991.

[50] R. Cespedes. New method for the analysis of distribution networks. IEEE Trans. Power Del., 5(1):391-396, January 1990.

[51] A. G. Expósito and E. R. Ramos. Reliable load flow technique for radial distribution networks. IEEE Trans. Power Syst., 14(13):10631069, August 1999.

[52] Joshua A. Taylor and Franz S. Hover. Convex models of distribution system reconfiguration. IEEE Trans. Power Systems, 2012.

[53] J. Lavaei, D. Tse, and B. Zhang. Geometry of power flows and optimization in distribution networks. Power Systems, IEEE Transactions on, PP(99):1-12, 2013.

[54] W. H. Kersting. Distribution systems modeling and analysis. CRC, 2002.

[55] D. Shirmohammadi, H. W. Hong, A. Semlyen, and G. X. Luo. A compensation-based power flow method for weakly meshed distribution and transmission networks. IEEE Transactions on Power Systems, 3(2):753-762, May 1988.

[56] Lingwen Gan, Na Li, Ufuk Topcu, and Steven H. Low. Exact convex relaxation of optimal power flow in radial networks. IEEE Trans. Automatic Control, 2014.

[57] Subhonmesh Bose, Steven H. Low, and Mani Chandy. Equivalence of branch flow and bus injection models. In 50th Annual Allerton Conference on Communication, Control, and Computing, October 2012.

[58] Deqiang Gan, Robert J. Thomas, and Ray D. Zimmerman. Stability-constrained optimal power flow. IEEE Trans. Power Systems, 15(2):535-540, May 2000. 
[59] F. Capitanescua, J.L. Martinez Ramosb, P. Panciaticic, D. Kirschend, A. Marano Marcolinie, L. Platbroodf, and L. Wehenkelg. Stateof-the-art, challenges, and future trends in security constrained optimal power flow. Electric Power Systems Research, 81(8):1731-1741, August 2011.

[60] Brian Stott and Ongun Alsaç. Optimal power flow: Basic requirements for real-life problems and their solutions. White paper, July 2012.

[61] S. Bose, D. Gayme, K. M. Chandy, and S. H. Low. Quadratically constrained quadratic programs on acyclic graphs with application to power flow. arXiv:1203.5599v1, March 2012.

[62] R. Grone, C. R. Johnson, E. M. Sá, and H. Wolkowicz. Positive definite completions of partial Hermitian matrices. Linear Algebra and its Applications, 58:109-124, 1984.

[63] Mituhiro Fukuda, Masakazu Kojima, Kazuo Murota, and Kazuhide Nakata. Exploiting sparsity in semidefinite programming via matrix completion I: General framework. SIAM Journal on Optimization, 11:647-674, 2001.

[64] K. Nakata, K. Fujisawa, M. Fukuda, M. Kojima, and K. Murota. Exploiting sparsity in semidefinite programming via matrix completion II: Implementation and numerical results. Mathematical Programming, 95(2):303-327, 2003.

[65] Baosen Zhang and David Tse. Geometry of the injection region of power networks. IEEE Trans. Power Systems, 28(2):788-797, 2013.

[66] Martin S. Andersen, Anders Hansson, and Lieven Vandenberghe. Reduced-complexity semidefinite relaxations of optimal power flow problems. arXiv:1308.6718v1, August 2013.

[67] R.D. Zimmerman and Hsiao-Dong Chiang. Fast decoupled power flow for unbalanced radial distribution systems. Power Systems, IEEE Transactions on, 10(4):2045-2052, 1995.

[68] M.S. Srinivas. Distribution load flows: a brief review. In Power Engineering Society Winter Meeting, 2000. IEEE, volume 2, pages 942-945 vol.2, 2000.

[69] M.E. Baran and F.F. Wu. Network reconfiguration in distribution systems for loss reduction and load balancing. IEEE Trans. on Power Delivery, 4(2):1401-1407, Apr 1989.

[70] S. P. Boyd and L. Vandenberghe. Convex optimization. Cambridge University Press, 2004.

[71] H. Wolkowicz, R. Saigal, and L. Vandenberghe. Handbook of semidefinite programming: theory, algorithms, and applications, volume 27. Springer Netherlands, 2000.

[72] Miguel Soma Lobo, Lieven Vandenberghe, Stephen Boyd, and Hervé Lebret. Applications of second-order cone programming. Linear Algebra and its Applications, 284:193-228, 1998.

[73] S. Zhang. Quadratic maximization and semidefinite relaxation. Mathematical Programming, 87(3):453-465, 2000.

[74] S. Kim and M. Kojima. Exact solutions of some nonconvex quadratic optimization problems via SDP and SOCP relaxations. Computational Optimization and Applications, 26(2):143-154, 2003.

[75] D. R. Fulkerson and O. A. Gross. Incidence matrices and interval graphs. Pacific Journal of Mathematics, 15(3):835-855, 1965.

[76] Donald J. Rose, Robert Endre Tarjan, and George S. Lueker. Algorithmic aspects of vertex elimination on graphs. SIAM Journal on Computing, 5(2):266-283, 1976.

[77] Norman Biggs. Algebraic graph theory. Cambridge University Press, 1993. Cambridge Mathematical Library. 